\title{
Synthesis of the First Stable Palladium Allenylidene Complexes
}

\author{
Florian Kessler, Normen Szesni, Kaija Põhako, Bernhard Weibert, and Helmut Fischer* \\ Fachbereich Chemie, Universität Konstanz, Fach 727, 78457 Konstanz, Germany
}

\begin{abstract}
Oxidative addition of $\mathrm{BrC} \equiv \mathrm{CC}(=\mathrm{O}) \mathrm{NR}_{2}$ to $\left[\mathrm{Pd}\left(\mathrm{PPh}_{3}\right)_{4}\right]$ affords the trans-alkynylbromopalladium complexes trans- $\left[\mathrm{Br}\left(\mathrm{PPh}_{3}\right)_{2} \mathrm{Pd}-\mathrm{C} \equiv \mathrm{CC}(=\mathrm{O}) \mathrm{NR}_{2}\right]\left(\mathrm{NR}_{2}=\mathrm{NMe}_{2}(\mathbf{2 a}), \mathrm{N}\left(\mathrm{CH}_{2}\right)_{4}\right)$ (2b)). Subsequent reaction of $\mathbf{2 a}, \mathbf{b}$ with $\mathrm{P}^{i} \mathrm{Pr}_{3}$ in excess gives trans- $\left[\mathrm{Br}\left(\mathrm{P}^{i} \mathrm{Pr}_{3}\right)_{2} \mathrm{Pd}-\mathrm{C} \equiv \mathrm{CC}(=\mathrm{O}) \mathrm{NR}_{2}\right](\mathbf{5 a}, \mathbf{b})$. The analogous reaction of $2 \mathbf{b}$ with $\mathrm{P}\left(\mathrm{C}_{6} \mathrm{H}_{4} \mathrm{OMe}-4\right)_{3}$ gives trans- $\left[\mathrm{Br}\left(\mathrm{P}\left\{\mathrm{C}_{6} \mathrm{H}_{4} \mathrm{OMe}-4\right\}_{3}\right)_{2} \mathrm{Pd}-\mathrm{C} \equiv \mathrm{CC}(=\mathrm{O}) \mathrm{NR}_{2}\right](\mathbf{7 b})$, and that of $\mathbf{2 a}$ with trifluoroacetate gives trans $-\left[\left(\mathrm{F}_{3} \mathrm{CCOO}\right)\left(\mathrm{PPh}_{3}\right)_{2} \mathrm{Pd}-\mathrm{C} \equiv \mathrm{CC}(=\mathrm{O}) \mathrm{NMe}_{2}\right](\mathbf{9 a})$. Methylation of 2a, $\mathbf{b}$, $\mathbf{7 b}$, and $\mathbf{9 a}$ with either MeOTf or $\left[\mathrm{Me}_{3} \mathrm{O}\right] \mathrm{BF}_{4}$ and ethylation of $\mathbf{2 a}, \mathbf{b}$ with $\left[\mathrm{Et}_{3} \mathrm{O}\right] \mathrm{BF}_{4}$ yield the first cationic allenylidene complexes of palladium, trans- $\left[\mathrm{R}^{*}\left(\mathrm{PR}_{3}^{\prime}\right)_{2} \mathrm{Pd}-\mathrm{C} \equiv \mathrm{CC}(\mathrm{OMe}) \mathrm{NR}_{2}\right]^{+} \mathrm{X}^{-}\left(\mathrm{R}^{*}=\mathrm{Br}, \mathrm{CF}_{3} \mathrm{COO}\right.$; $\left.\mathrm{R}^{\prime}=\mathrm{Ph}, \mathrm{C}_{6} \mathrm{H}_{4} \mathrm{OMe}-4,{ }^{i} \mathrm{Pr} ; \mathrm{X}=\mathrm{OTf}, \mathrm{BF}_{4}\right)$.
\end{abstract}

\section{Introduction}

The synthesis of the first allenylidene complexes, $\mathrm{L}_{n} \mathrm{M}=\mathrm{C}=\mathrm{C}=\mathrm{C}\left(\mathrm{R}^{1}\right) \mathrm{R}^{2}$, was reported in 1976 simultaneously by Fischer et al. $(\mathrm{M}=\mathrm{Cr}, \mathrm{W})^{1}$ and Berke $(\mathrm{M}=\mathrm{Mn}){ }^{2}$ Fischer's $^{2}$ synthesis involved Lewis acid induced ethanol abstraction from ethoxycarbene complexes $\left[(\mathrm{CO})_{5} \mathrm{M}=\mathrm{C}(\mathrm{OEt})(\mathrm{CH}=\mathrm{C}\right.$ $\left.\left.\left(\mathrm{NMe}_{2}\right) \mathrm{Ph}\right)\right]$. Berke obtained the manganese allenylidene complex $\left[\mathrm{Cp}(\mathrm{CO})_{2} \mathrm{Mn}=\mathrm{C}=\mathrm{C}=\mathrm{C}\left({ }^{\mathrm{t}} \mathrm{Bu}\right)_{2}\right]$ on treatment of the methyl propiolate complex $\left[\mathrm{Cp}(\mathrm{CO})_{2} \mathrm{Mn}(\mathrm{HC} \equiv \mathrm{CCOOMe})\right]$ with an excess of ${ }^{\mathrm{t}} \mathrm{BuLi}$, presumably via an alkynyl complex as an intermediate. Since then a large number of allenylidene complexes of many transition metals have been prepared, including complexes of titanium, chromium, tungsten, manganese, rhenium, iron, ruthenium, osmium, rhodium, and iridium. ${ }^{3}$ Most syntheses now use propargylic alcohols, $\mathrm{HC} \equiv \mathrm{CC}(\mathrm{R})\left(\mathrm{R}^{\prime}\right) \mathrm{OH}$, as sources of the allenylidene $\mathrm{C}_{3}$ fragment. Coordination of the propargylic alcohol to the transition metal is followed by its rearrangement into a hydroxyvinylidene ligand. On subsequent elimination of water, allenylidene ligands are formed. This strategy was originally introduced by Selegue. ${ }^{4}$ Some of these complexes have been used as catalyst precursors: $:^{5}$ for instance,

* To whom correspondence should be addressed. E-mail: helmut. fischer@uni-konstanz.de. Fax: +7531-883136.

(1) Fischer, E. O.; Kalder, H. J.; Frank, A.; Köhler, F. H.; Huttner, G. Angew. Chem. 1976, 88, 683; Angew. Chem., Int. Ed. Engl. 1976, 15, 623.

(2) Berke, H. Angew. Chem. 1976, 88, 684; Angew. Chem., Int. Ed. Engl. 1976, 15,624 .

(3) For reviews see: (a) Bruce, M. I.; Swincer, A. G. Adv. Organomet. Chem. 1983, 22, 59. (b) Bruce, M. I. Chem. Rev. 1991, 91, 197. (c) Doherty, S.; Corrigan, J. F.; Carty, A. J.; Sappa, E. Adv. Organomet. Chem. 1995, 37, 39. (d) Werner, H. J. Chem. Soc., Chem. Commun. 1997, 903. (e) Bruce M. I. Chem. Rev. 1998, 98, 2797. (f) Touchard, D.; Dixneuf, P. H. Coord. Chem. Rev. 1998, 178-180, 409. (g) Cadierno, V.; Gamasa, M. P.; Gimeno, J. Eur. J. Inorg. Chem. 2001, 571. (h) Winter, R. F.; Zális, S. Coord. Chem. Rev. 2004, 248, 1565. (i) Rigaut, S.; Touchard, D.; Dixneuf, P. H. Coord. Chem. Rev. 2004, 248, 1585. (j) Cadierno, V.; Gamasa, M. P.; Gimeno, J. Coord. Chem. Rev. 2004, 248, 1627. (k) Cadierno, V.; Crochet, P.; Gimeno, J. In Metal Vinylidenes and Allenylidenes in Catalysis; Bruneau, C., Dixneuf, P. H., Eds.; Wiley-VCH: Weinheim, Germany, 2008; p $61 \mathrm{ff}$.

(4) Selegue, J. P. Organometallics 1982, 1, 217.

(5) For recent reviews see: (a) Castarlenas, R ; Fischmeister, C.; Bruneau, C.; Dixneuf, P. H. J. Mol. Catal. A 2004, 213, 31. (b) Bruneau, C.; Dixneuf, P. H. Angew. Chem. 2006, 118, 2232; Angew. Chem., Int. Ed. 2006, 45, 2176. in ring-closing metathesis, ${ }^{6}$ in ring-opening metathesis, ${ }^{7}$ in the dehydrogenative dimerization of tin hydrides, ${ }^{8}$ and in selective transetherification of substituted vinyl ethers. ${ }^{9}$

Allenylidene complexes of palladium have been unknown until now, and consequently their catalytic activity has not been studied. This is surprising, especially when considering the broad range of applications of palladium complexes in organic synthesis and catalysis. ${ }^{10}$ Many commonly used catalysts for $\mathrm{CC}$ coupling reactions such as, for example, the Mizoroki-Heck reaction or the Suzuki coupling reaction are based on palladium complexes.

We now report on the synthesis and the spectroscopic properties of the first palladium allenylidene complexes from readily available $N, N$-dimethylpropiolamides as the $\mathrm{C}_{3}$ source.

\section{Results and Discussion}

Initially, we envisioned the transmetalation of allenylidene ligands from chromium to palladium, since $\mathrm{N}$-heterocyclic carbene ligands such as pyrazolin-3-ylidene and pyrazolidin-

(6) (a) Fürstner, A.; Picquet, M.; Bruneau, C.; Dixneuf, P. H. Chem. Commun. 1998, 1315. (b) Picquet, M.; Touchard, D.; Bruneau, C.; Dixneuf, P. H. New J. Chem. 1999, 23, 141. (c) Osipov, S. N.; Artyushin, O. I.; Kolomiets, A. F.; Bruneau, C.; Picquet, M.; Dixneuf, P. H. Eur. J. Org. Chem. 2001, 3891. (d) Picquet, M.; Bruneau, C.; Dixneuf, P. H. Chem. Commun. 1998, 2249. (e) Sémeril, D.; Le Nôtre, J.; Bruneau, C.; Dixneuf, P. H.; Kolomiets, A. F.; Osipov, S. N. New J. Chem. 2001, 25, 16. (f) Schanz, H.-J.; Jafarpour, L.; Stevens, E. D.; Nolan, S. P. Organometallics 1999, 18, 5187. (g) Jafarpour, L.; Huang, J.; Stevens, E. D.; Nolan, S. P. Organometallics 1999, 18, 3760. (h) Le Gendre, P.; Picquet, M.; Richard, P.; Moise, C. J. Organomet. Chem. 2002, 643-644, 231. (i) Fürstner, A.; Müller, T. J. Am. Chem. Soc. 1999, 121, 7814. (j) Fürstner, A.; Thiel, O. R. J. Org. Chem. 2000, 65, 1738. (k) Özdemir, I.; Cetinkaya, E.; Cetinkaya, B.; Cicek, M.; Sémeril, D.; Bruneau, C.; Dixneuf, P. H. Eur. J. Inorg. Chem. 2004, 418. (1) Akiyama, R.; Kobayashi, S. Angew. Chem. 2002, 114, 2714; Angew. Chem., Int. Ed. 2002, 41, 2602.

(7) (a) Saoud, M.; Romerosa, A.; Peruzzini, M. Organometallics 2000 , 19, 4005. (b) Castarlenas, R.; Sémeril, D.; Noels, A. F.; Demonceau, A.; Dixneuf, P. H. J. Organomet. Chem. 2002, 663, 235. (c) Alaoui Abdallaoui, I.; Sémeril, D.; Dixneuf, P. H. J. Mol. Catal. A 2002, 182-183, 577.

(8) Maddock, S. M.; Finn, M. G. Angew. Chem. 2001, 113, 2196; Angew. Chem., Int. Ed. 2001, 40, 2138

(9) Saoud, M.; Romerosa, A.; Manas Carpio, S.; Gonsalvi, L.; Peruzzini, M. Eur. J. Inorg. Chem. 2003, 1614.

(10) See e.g.: (a) Heck, R. F. Palladium Reagents in Organic Synthesis; Academic Press: London, 1985. (b) de Meijere, A., Diederich, F. Eds. MetalCatalyzed Cross-Coupling Reactions; Wiley-VCH: Weinheim, Germany, 2004. (c) Tsuji, J. Palladium Reagents and Catalysis; Wiley: Chichester, U.K., 2004. 


\section{Scheme 1}

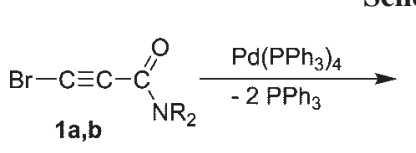

$\mathrm{PPh}_{3}$
$\mathrm{Br}-\mathrm{Pd}-\mathrm{C} \equiv \mathrm{C}-\mathrm{C}^{\prime}$
$\mathrm{PPh}_{3}$

$2 a, b$

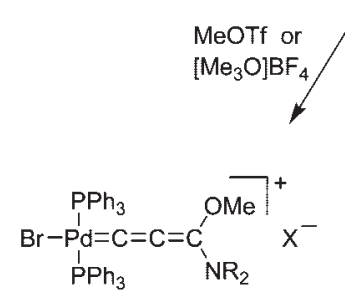

$3 a-X, 3 b-X$

$\mathrm{NR}_{2}=\mathrm{NMe}_{2}(\mathrm{a}), \mathrm{N}$ (b) $\mathrm{X}=\mathrm{OTf}, \mathrm{BF}_{4}$

3-ylidene proved readily transferable from pentacarbonylchromium complexes to gold, palladium, and platinum in high yield. ${ }^{11}$ The analogous transmetalation of several allenylidene ligands from chromium to tungsten likewise proceeded quickly in yields ranging from 83 to $97 \% .^{12}$ However, all attempts to transfer allenylidene ligands from chromium to palladium met with failure. Therefore, the strategy had to be changed and an approach starting from alkynyl complexes was investigated.

Recently, we developed an easy to perform one-pot synthesis for $\pi$-donor-substituted allenylidene pentacarbonyl complexes of chromium and tungsten. Sequential reaction of the solvent complexes $\left[(\mathrm{CO})_{5} \mathrm{M}(\mathrm{THF})\right](\mathrm{M}=\mathrm{Cr}, \mathrm{W})$ with appropriate deprotonated alkynes as the $\mathrm{C}_{3}$ source and $\left[\mathrm{R}_{3} \mathrm{O}\right] \mathrm{BF}_{4}$ as the alkylating agent afforded the corresponding amino- and alkoxyallenylidene complexes in very good yields. ${ }^{13}$ Modification of this route turned out to also be applicable to the preparation of palladium allenylidene complexes.

Terminal halogenoalkynes are known to react with zerovalent palladium complexes by oxidative addition, affording stable palladium(II) alkynyl complexes. ${ }^{14}$ Thus, treatment of a suspension of $\left[\mathrm{Pd}\left(\mathrm{PPh}_{3}\right)_{4}\right]$ in $\mathrm{CH}_{2} \mathrm{Cl}_{2}$ at ambient temperature with $\mathrm{BrC} \equiv \mathrm{CC}(=\mathrm{O}) \mathrm{NMe}_{2}(\mathbf{1 a})$ afforded the neutral alkynyl complex 2a (Scheme 1). Bromoalkyne 1a was obtained by reaction of propynoic acid dimethylamide with $N$-bromosuccinimide (NBS). Pure alkynyl complex 2a was isolated, after repeated crystallization from mixtures of $\mathrm{CH}_{2} \mathrm{Cl}_{2}$ and $\mathrm{Et}_{2} \mathrm{O}$, as a colorless solid in $85 \%$ yield. The subsequent alkylation of $2 \mathrm{a}$ at $-50{ }^{\circ} \mathrm{C}$ with a slight excess of MeOTf proceeded smoothly and afforded the cationic palladium allenylidene complex 3a-OTf as a light yellow solid in $91 \%$ yield after crystallization from pentane$\mathrm{CH}_{2} \mathrm{Cl}_{2}$ mixtures (Scheme 1). The corresponding $\mathrm{BF}_{4}$ salt, $\mathbf{3 a}$ $\mathrm{BF}_{4}$, was obtained when $\left[\mathrm{Me}_{3} \mathrm{O}\right] \mathrm{BF}_{4}$ instead of MeOTf was used as the alkylation agent. The complexes $\mathbf{2 b}, \mathbf{3 b}-\mathrm{OTf}, \mathbf{3} \mathbf{b}-\mathrm{BF}_{4}$, $\mathbf{4 a}-\mathrm{BF}_{4}$, and $\mathbf{4 b}-\mathrm{BF}_{4}$ were synthesized accordingly.

(11) Kessler, F.; Szesni, N.; Maass, C.; Hohberger, C.; Weibert, B.; Fischer, H. J. Organomet. Chem. 2007, 692, 3005.

(12) Szesni, N.; Drexler, M.; Fischer, H. Organometallics 2006, 25, 3989.

(13) (a) Fischer, H.; Szesni, N.; Roth, G.; Burzlaff, N.; Weibert, B. J. Organomet. Chem. 2003, 683, 301. (b) Fischer, H ; Szesni, N. Coord. Chem. Rev. 2004, 248, 1659.

(14) (a) Burgess, J.; Howden, M. E.; Kemmitt, R. D. W.; Sridhara, N. S. J. Chem. Soc., Dalton Trans. 1978, 1577. (b) Klein, H. F.; Zettel, B. D. Chem. Ber. 1995, 128, 343. (c) Mann, G.; Baranano, D.; Hartwig, J. F.; Rheingold, A. L.; Guzai, I. A. J. Am. Chem. Soc. 1998, 120, 9205. (d) Weigelt, M.; Becher, D.; Poetsch, E.; Bruhn, C.; Steinborn, D. Z. Anorg. Allg. Chem. 1999, 625, 1542. (e) Osakada, K.; Hamada, M.; Yamamoto, T. Organometallics 2000, 19, 458.

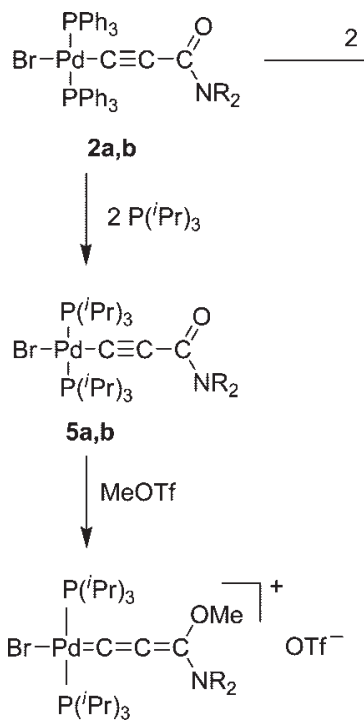

6a-OTf, 6b-OTf
Scheme 2

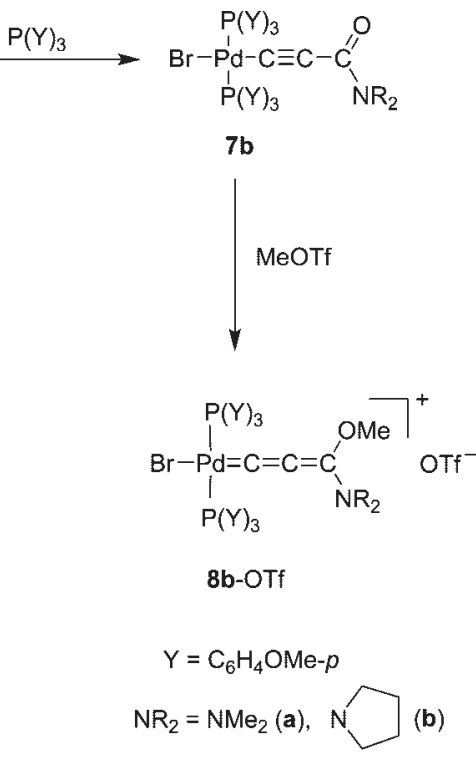

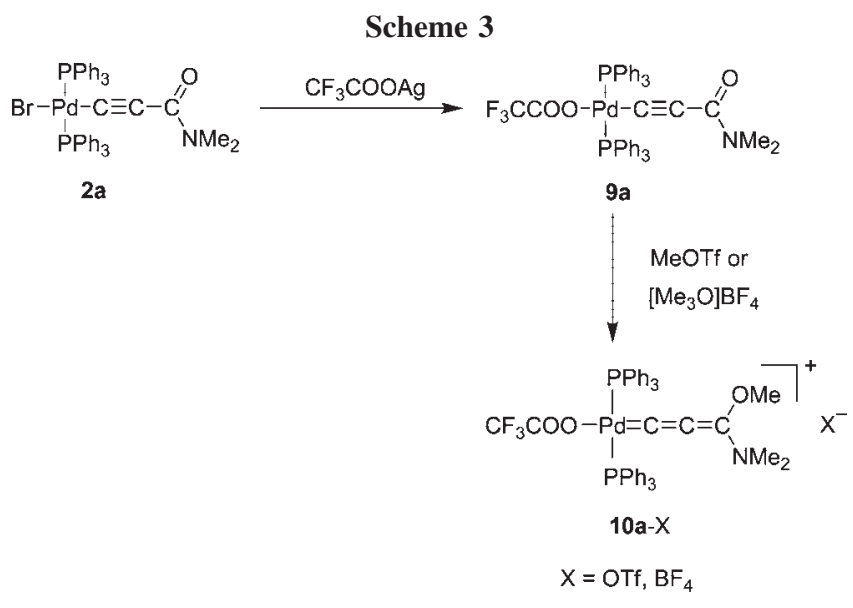

Modification of the properties of the allenylidene complex can be achieved by variation of the terminal substituents of the allenylidene ligand and the coligands at palladium. The variation of the terminal substituents was achieved by starting from alkyne $\mathbf{1 b}$ instead of $\mathbf{1 a}$ and employing $\left[\mathrm{Et}_{3} \mathrm{O}\right] \mathrm{BF}_{4}$ as the alkylation agent; otherwise the same reaction sequence was followed (Scheme 1).

The metal-bound $\mathrm{C}_{\alpha}$ atom and the terminal $\mathrm{C}_{\gamma}$ atom in allenylidene complexes are electrophilic centers (see resonance forms II and III in Scheme 4). ${ }^{3}$ Therefore, nucleophilic additions to these centers might compete with substitution of halides for the bromide ligand or of phosphines for the $\mathrm{PPh}_{3}$ ligands. To avoid such side reactions at the allenylidene ligand, the alkynyl complexes 2a,b were chosen as the starting compounds for the modification of the coligand set in allenylidene complexes.

Treatment of a solution of $\mathbf{2 a}, \mathbf{b}$ in $\mathrm{CH}_{2} \mathrm{Cl}_{2}$ with 2.2 equiv of the more nucleophilic phosphines $\mathrm{P}^{i} \mathrm{Pr}_{3}$ and $\mathrm{P}\left(\mathrm{C}_{6} \mathrm{H}_{4} \mathrm{OMe}-4\right)_{3}$ led to quantitative exchange of both $\mathrm{PPh}_{3}$ ligands. Complexes $\mathbf{5 a}, \mathbf{b}$ and $\mathbf{7 b}$ were obtained as colorless or pale yellow solids after several recrystallization cycles from $\mathrm{Et}_{2} \mathrm{O}$ in $70-74 \%$ yield. These alkynyl complexes were subsequently converted into cationic allenylidene complexes by alkylation with MeOTf. The resulting allenylidene complexes were then isolated in $98 \%$ (6aOTf), 97\% (6b-OTf), and 91\% yield (8b-OTf) (Scheme 2).

When $\mathrm{Ag}^{+}\left[\mathrm{CF}_{3} \mathrm{COO}\right]^{-}$was added to a solution of $\mathbf{2 a}$ in $\mathrm{CH}_{2} \mathrm{Cl}_{2}, \mathrm{AgBr}$ instantaneously precipitated and the trifluoroac- 


\section{Scheme 4}



etato complex 9a was isolated as a colorless solid in $96 \%$ yield. Alkylation of 9a with MeOTf or $\left[\mathrm{Me}_{3} \mathrm{O}\right] \mathrm{BF}_{4}$ finally afforded the allenylidene complexes 10a-OTf and $\mathbf{1 0 a}-\mathrm{BF}_{4}$ in $90 \%$ and $86 \%$ yields, respectively (Scheme 3 ).

All new alkynyl and allenylidene complexes were characterized by spectroscopic means and by elemental analysis. The structures of $\mathbf{5 b}$ and $\mathbf{1 0 a}-\mathrm{BF}_{4}$ were additionally established by $\mathrm{X}$-ray diffraction studies.

From the observation of only one singlet in the ${ }^{31} \mathrm{P}$ NMR spectra it followed that the two phosphine ligands are mutually trans. There was no indication of the presence of a cis isomer. Two singlets for the two N-bound methyl groups in the NMR spectra of all alkynyl complexes indicated a rather high barrier to rotation around the $\mathrm{C}\left(\mathrm{sp}^{2}\right)-\mathrm{N}$ bond. From the coalescence of the two signals of complex $2 \mathbf{a}$ in $\mathrm{C}_{2} \mathrm{D}_{2} \mathrm{Cl}_{4}$ at $115^{\circ} \mathrm{C}$ an energy barrier of $\Delta G^{\ddagger}=76.1 \pm 0.4 \mathrm{~kJ} / \mathrm{mol}$ was calculated. The barrier is slightly lower than that in free propiolamides $(\mathrm{RC} \equiv \mathrm{CC}$ $\left.(=\mathrm{O}) \mathrm{NMe}_{2}, \mathrm{R}=\mathrm{H}, \mathrm{Me}, \mathrm{Ph}: 79.6-82.1 \mathrm{kcal} / \mathrm{mol}\right),{ }^{15}$ indicating minor back-donation from palladium to the alkynyl ligand and almost negligible interaction of the metal with the $\mathrm{C}(=\mathrm{O}) \mathrm{NMe}_{2}$ fragment. The resonances of the alkynyl ligand in the ${ }^{13} \mathrm{C} N M R$ spectra compared well with those of known palladium alkynyl complexes. ${ }^{14 \mathrm{~d}}$ As expected, increasing the electron density at palladium in the series $\mathbf{9 a}, \mathbf{2} \mathbf{a}, \mathbf{7} \mathbf{b}$ led to a shift of the ${ }^{13} \mathrm{C}$ resonance of the metal-bound alkynyl $\mathrm{C}_{\alpha}$ atom to lower field. The resonances of $\mathrm{C}_{\beta}$ and $\mathrm{C}_{\gamma}$ were unaffected by varying the substitution pattern of the metal center.

The formation of the cationic allenylidene complexes by alkylation of the alkynyl complexes was accompanied by a pronounced shift of the $\mathrm{C}_{\alpha}$ resonance to lower field by about $45 \mathrm{ppm}$, a shift of the $\mathrm{C}_{\beta}$ resonance to higher field $(\Delta \delta \approx 11$ $\mathrm{ppm})$ and a shift of the $v(\mathrm{CC})$ vibration to lower energy by $10-15 \mathrm{~cm}^{-1}$. The resonances of the $\mathrm{C}_{\gamma}$ atom and the $\mathrm{N}-\mathrm{CH}_{3}$ groups were again almost unaffected by the alkylation. Similar trends have been observed on alkylation of alkynylpentacarbonylchromate complexes to give neutral allenylidene complexes. ${ }^{12}$ The extent of these shifts and the observation of two resonances for the dimethylamino substituent in the ${ }^{1} \mathrm{H}$ and ${ }^{13} \mathrm{C} N M R$ spectra demonstrate the importance of the zwitterionic resonance forms II and III for the overall bond description of these cationic allenylidene complexes (Scheme 4). ${ }^{12}$ As in $\mathbf{2 a}, \mathbf{5 a}, \mathbf{7 b}$, and 9a, both phosphine ligands are mutually trans, as indicated by the presence of only one signal for both phosphorus nuclei in the ${ }^{31} \mathrm{P}$ NMR spectra.

A comparison of the spectroscopic data of these cationic palladium allenylidene complexes with those of the related neutral complexes $\left[(\mathrm{CO})_{5} \mathrm{M}=\mathrm{C}=\mathrm{C}=\mathrm{C}\left(\mathrm{NMe}_{2}\right) \mathrm{OMe}\right](\mathrm{M}=\mathrm{Cr}$, $\mathrm{W})$ reveals that in cationic palladium allenylidene complexes the alkynyl character (see III in Scheme 4) is significantly more pronounced than in the corresponding group 6 complexes, as evidenced by the $v(\mathrm{CC})$ vibration at higher energy by about $70-90 \mathrm{~cm}^{-1}$.

The solid-state structures of the alkynyl complex $\mathbf{5 b}$ (Figure 1) and of the cationic allenylidene complex $\mathbf{1 0 a}-\mathrm{BF}_{4}$ (Figure 2)

(15) (a) Oki, M. Applications of Dynamic NMR Spectroscopy to Organic Chemistry; VCH: Deerfield Beach, FL, 1985. (b) Jackman, L. M., Cotton, F. A., Eds. Dynamic Nuclear Magnetic Resonance Spectroscopy; Academic Press: New York, 1975.

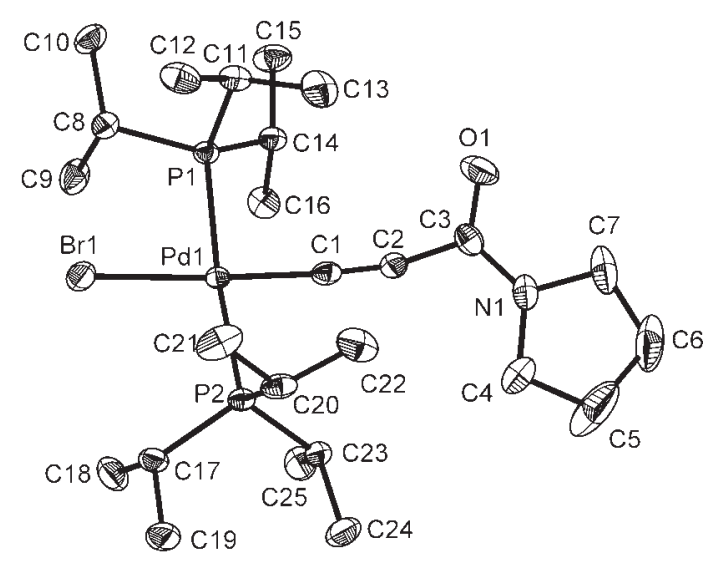

Figure 1. Structure of the alkynyl complex $\mathbf{5 b}$ in the crystal (ellipsoids drawn at the $50 \%$ probability level; hydrogen atoms omitted for clarity). Important distances $(\AA)$ and angles (deg): $\mathrm{Pd}(1)-\mathrm{C}(1)=1.947(3), \operatorname{Pd}(1)-\mathrm{Br}(1)=2.4629(8), \operatorname{Pd}(1)-\mathrm{P}(1)=$ 2.3581(9), $\mathrm{Pd}(1)-\mathrm{P}(2)=2.3500(9), \mathrm{C}(1)-\mathrm{C}(2)=1.209(5)$, $\mathrm{C}(2)-\mathrm{C}(3)=1.454(4), \mathrm{C}(3)-\mathrm{O}(1)=1.244(4), \mathrm{C}(3)-\mathrm{N}(1)=$ $1.331(4), \mathrm{N}(1)-\mathrm{C}(4)=1.466(5) ; \mathrm{C}(1)-\mathrm{Pd}(1)-\mathrm{Br}(1)=176.41(9)$, $\mathrm{Pd}(1)-\mathrm{C}(1)-\mathrm{C}(2)=175.6(3), \mathrm{C}(1)-\mathrm{C}(2)-\mathrm{C}(3)=168.1(3)$, $\mathrm{C}(2)-\mathrm{C}(3)-\mathrm{O}(1)=120.5(3), \mathrm{C}(2)-\mathrm{C}(3)-\mathrm{N}(1)=117.5(3)$.

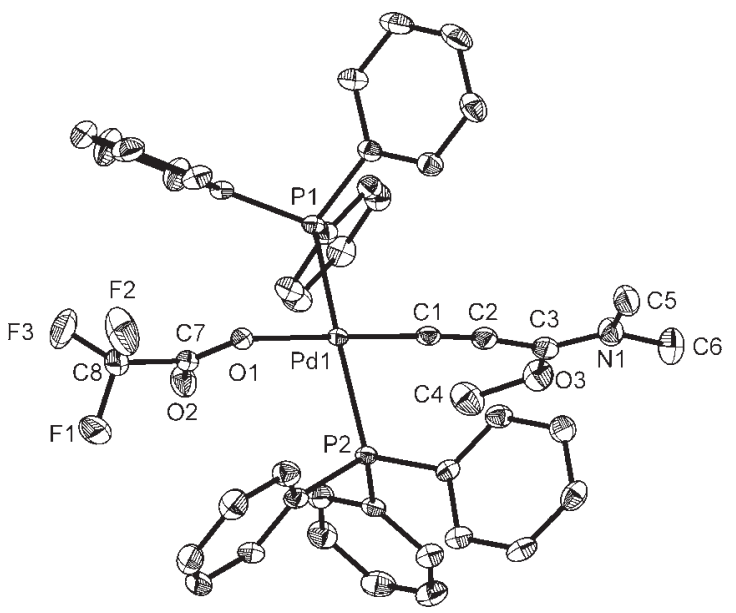

Figure 2. Structure of the cation of complex $\mathbf{1 0 a}-\mathrm{BF}_{4}$ in the crystal (ellipsoids drawn at the $50 \%$ probability level; hydrogen atoms, two molecules of methylene chloride, and the anion $\mathrm{BF}_{4}{ }^{-}$omitted for clarity). Important distances $(\AA)$ and angles (deg): $\mathrm{Pd}(1)-\mathrm{C}(1)$ $=1.925(3), \mathrm{Pd}(1)-\mathrm{O}(1)=2.067(2), \mathrm{Pd}(1)-\mathrm{P}(1)=2.3338(9)$, $\mathrm{Pd}(1)-\mathrm{P}(2)=2.3303(9), \mathrm{C}(1)-\mathrm{C}(2)=1.217(4), \mathrm{C}(2)-\mathrm{C}(3)=$ $1.420(4), \mathrm{C}(3)-\mathrm{N}(1)=1.296(4), \mathrm{C}(3)-\mathrm{O}(3)=1.330(4), \mathrm{O}(3)-\mathrm{C}(4)$ $=1.446(4), \mathrm{O}(1)-\mathrm{C}(7)=1.267(3), \mathrm{O}(2)-\mathrm{C}(7)=1.223(4)$; $\mathrm{C}(1)-\mathrm{Pd}(1)-\mathrm{O}(1)=178.69(9), \mathrm{Pd}(1)-\mathrm{C}(1)-\mathrm{C}(2)=176.7(2)$, $\mathrm{C}(1)-\mathrm{C}(2)-\mathrm{C}(3)=172.6(3), \mathrm{C}(2)-\mathrm{C}(3)-\mathrm{N}(1)=123.0(3)$, $\mathrm{C}(2)-\mathrm{C}(3)-\mathrm{O}(3)=120.8(3), \mathrm{N}(1)-\mathrm{C}(3)-\mathrm{O}(3)=116.2(3)$.

were determined by X-ray diffraction studies. The complex 10a$\mathrm{BF}_{4}$ crystallizes from dichloromethane with two molecules of $\mathrm{CH}_{2} \mathrm{Cl}_{2}$; the $\mathrm{BF}_{4}^{-}$anion is slightly disordered. In both complexes the palladium atom engages in square-planar coordination. In $\mathbf{5 b}$ the plane formed by the atoms $\mathrm{C}(3), \mathrm{O}(1)$, and $\mathrm{N}(1)$ and the coordination plane of palladium are almost coplanar (torsion angle $\left.\mathrm{O}(1)-\mathrm{C}(3)-\mathrm{Pd}(1)-\mathrm{P}(1)=12.0^{\circ}\right)$. In contrast, the allenylidene plane (formed by the atoms $\mathrm{C}(3), \mathrm{N}(1)$, and $\mathrm{O}(3)$ ) and the trifluoracetate plane in $\mathbf{1 0 a}-\mathrm{BF}_{4}$ are perpendicular $(89.6$ and $87.9^{\circ}$, respectively) to the coordination plane of palladium. In both complexes the $\mathrm{Pd}-\mathrm{C}_{3}$ chain is slightly bent: $\mathrm{Pd}-\mathrm{C}(1)-\mathrm{C}(2)$ $=175.6(3)^{\circ}(\mathbf{5 b})$ and $176.7(2)^{\circ}\left(\mathbf{1 0 a}-\mathrm{BF}_{4}\right), \mathrm{C}(1)-\mathrm{C}(2)-\mathrm{C}(3)$ $=168.1(3)^{\circ}(\mathbf{5 b})$ and $172.6(3)^{\circ}\left(\mathbf{1 0 a}-\mathrm{BF}_{4}\right)$. However, a modest 
deviation from linearity of the $\mathrm{MC}_{3}$ fragment in allenylidene complexes is often observed. ${ }^{3}$

The $\mathrm{Pd}-\mathrm{C}$ bond $(1.925(3) \AA$ ) in the allenylidene complex $10 \mathrm{a}-\mathrm{BF}_{4}$ is close to the shorter limit of observed $\mathrm{Pd}-\mathrm{C}$ bond lengths and is shorter than the $\mathrm{Pd}-\mathrm{C}$ bond in the related cationic $\mathrm{N}$-heterocyclic carbene (NHC) complexes trans- $\left[\mathrm{L}\left(\mathrm{PR}_{3}\right)_{2} \mathrm{Pd}-\right.$ $(\mathrm{NHC})]^{+}(1.97-2.01 \AA)^{16}$ or in the neutral alkynyl complexes trans- $\left[\mathrm{L}\left(\mathrm{PR}_{3}\right)_{2} \mathrm{Pd}-\mathrm{C} \equiv \mathrm{CR}\right](1.947(3) \AA$ in $\mathbf{5 b}$; range usually observed $1.95-2.07 \AA^{14 \mathrm{~d}, 17}$ ). Similarly to other neutral $\pi$-donorsubstituted allenylidene complexes of chromium and tung$\operatorname{sten}^{11-13,18}$ the $C(1)-C(2)$ bond is very short (1.217(4) $\AA$ ) and only slightly longer than in the alkynyl complex $\mathbf{5 b}(1.209(5)$ $\AA)$. Conversely, the $\mathrm{C}(2)-\mathrm{C}(3)$ bond in 10a- $\mathrm{BF}_{4}(1.420(4) \AA)$ is rather long and is even longer than that in $\left[(\mathrm{CO})_{5} \mathrm{Cr}=\mathrm{C}=\right.$ $\mathrm{C}=\mathrm{C}(\mathrm{O}$-adamantyl $\left.) \mathrm{NMe}_{2}\right](1.366(7) \AA)^{18}$ but, as expected, is shorter than in $\mathbf{5 b}$ (1.454(4) $\AA$ ). The terminal bonds of the chain, $\mathrm{C}(3)-\mathrm{O}(3)$ and $\mathrm{C}(3)-\mathrm{N}(3)$, however, compare well with those in related complexes.

In summary, the first isolable palladium allenylidene complexes are accessible by a straightforward two-step synthesis from readily available bromoalkynes. The new complexes are remarkably stable. For instance, after heating for $14 \mathrm{~h}$ at 160 ${ }^{\circ} \mathrm{C}$ the intensity of the $v(\mathrm{CC})$ vibration in the IR spectra of $\mathbf{3 a}$ OTf only decreased to a minor degree, thus confirming the stability of the new allenylidene complexes. They exhibit all characteristic features of $\pi$-donor-substituted allenylidene complexes.

\section{Experimental Section}

All reactions were performed under a nitrogen atmosphere by using standard Schlenk techniques. Solvents were dried by distillation from $\mathrm{CaH}_{2}\left(\mathrm{CH}_{2} \mathrm{Cl}_{2}\right), \mathrm{LiAlH}_{4}$ (pentane), and sodium $\left(\mathrm{Et}_{2} \mathrm{O}\right)$. The yields refer to analytically pure compounds and are not optimized. ${ }^{1} \mathrm{H},{ }^{13} \mathrm{C},{ }^{19} \mathrm{~F}$, and ${ }^{31} \mathrm{P}$ NMR spectra were recorded on a Jeol JNX 400, a Varian Inova 400, or a Bruker Avance 400 spectrometer at ambient temperature. Chemical shifts are reported relative to the residual solvent peaks or tetramethylsilane $\left({ }^{1} \mathrm{H},{ }^{13} \mathrm{C}\right)$ and $100 \% \mathrm{H}_{3} \mathrm{PO}_{4}\left({ }^{31} \mathrm{P}\right)$. Other instrumentation: IR, Biorad FTS 60; MS, Finnigan MAT 312; elemental analysis, Heraeus Elementar Vario EL. N,N-Dimethylpropiolamide ${ }^{19}$ and $\left[\mathrm{Pd}\left(\mathrm{PPh}_{3}\right)_{4}\right]^{20}$ were synthesized according to literature procedures. All other reagents were used as obtained from commercial suppliers.

1-Bromo- $N, N$-dimethylpropiolamide (1a). A solution of $0.97 \mathrm{~g}$ $(10 \mathrm{mmol})$ of $N, N$-dimethylpropiolamide in $40 \mathrm{~mL}$ of acetone was treated at ambient temperature with $2.16 \mathrm{~g}(12 \mathrm{mmol})$ of NBS and $150 \mathrm{mg}(0.9 \mathrm{mmol})$ of $\mathrm{AgNO}_{3}$. After $60 \mathrm{~min}$ the reaction mixture was poured onto $200 \mathrm{~mL}$ of ice water. The aqueous phase was extracted three times with $30 \mathrm{~mL}$ portions of ethyl acetate. The combined organic extracts were dried over $\mathrm{MgSO}_{4}$. The solid was then filtered off and the solvent removed in vacuo. The crude product was filtered over a short plug of silica using $\mathrm{CH}_{2} \mathrm{Cl}_{2}$ /acetone

(16) See e.g.: (a) Fürstner, A.; Seidel, G.; Kremzow, D.; Lehmann, C. W. Organometallics 2003, 22, 907. (b) Schneider, S. K.; Roembke, P.; Julius, G. R.; Raubenheimer, H. G.; Herrmann, W. A. Adv. Synth. Catal. 2006 348, 1862. (c) Schuster, O.; Raubenheimer, H. G. Inorg. Chem. 2006, 45, 7997.

(17) (a) Behrens, U.; Hoffmann, K. J. Organomet. Chem. 1977, 129, 273. (b) van der Voort, E.; Spek, A. L.; de Graaf, W. Acta Crystallogr., Sect. C: Cryst. Struct. Commun. 1987, 43, 2311. (c) Osakada, K.; Sakata, R.; Yamamoto, T. Organometallics 1997, 16, 5354. (d) Kim, Y.-J.; Lee, S.-H.; Lee, S.-H.; Jeon, S.-I.; Lim, M. S.; Lee, S. W. Inorg. Chim. Acta 2005, 358,650 617

(18) Szesni, N.; Weibert, B.; Fischer, H. Inorg. Chim. Acta 2006, 359,

(19) Crow, W. D.; Leonard, N. J. J. Org. Chem. 1965, 30, 2660.

(20) Tellier, F.; Sauvetre, R.; Normant, J. F. J. Organomet. Chem. 1985, 292,19
(5:1) as the eluant. Removal of the solvent gave $1.36 \mathrm{~g} \mathrm{(7.9} \mathrm{mmol;}$ $79 \%$ ) of 1 as a colorless solid. ${ }^{1} \mathrm{H}$ NMR $\left(400 \mathrm{MHz}, \mathrm{CDCl}_{3}\right): \delta$ $2.88\left(\mathrm{~s}, 3 \mathrm{H}, \mathrm{NCH}_{3}\right), 3.12\left(\mathrm{~s}, 3 \mathrm{H}, \mathrm{NCH}_{3}\right) \cdot{ }^{13} \mathrm{C} \mathrm{NMR}(100 \mathrm{MHz}$, $\left.\mathrm{CD}_{2} \mathrm{Cl}_{2}\right): \delta 34.0\left(\mathrm{NCH}_{3}\right), 38.0\left(\mathrm{NCH}_{3}\right), 55.2(\mathrm{C} \equiv C \mathrm{Br}), 73.4$ $(C \equiv \mathrm{CBr}), 153.0\left(C(\mathrm{O}) \mathrm{NMe}_{2}\right)$. IR $\left(\mathrm{CH}_{2} \mathrm{Cl}_{2}\right): v(\mathrm{C} \equiv \mathrm{C}) 2198 \mathrm{~cm}^{-1}$. EI-MS (70 eV): $m / z(\%) 176(48)\left[\mathrm{M}^{+}\right], 161(100)\left[\left(\mathrm{M}-\mathrm{CH}_{3}\right)^{+}\right]$. Anal. Calcd for $\mathrm{C}_{5} \mathrm{H}_{6} \mathrm{BrNO}$ (176.01): C, 34.12; H, 3.44; N, 7.96. Found: C, 34.09; H, 3.51; N, 8.05.

1-Bromo- $N, N$-tetramethylenepropiolamide (1b). The synthesis of $\mathbf{1 b}$ was carried out analogously to $\mathbf{1 a}$. The crude product was purified by column chromatography using $\mathrm{CH}_{2} \mathrm{Cl}_{2}$ as solvent. Yield: $1.72 \mathrm{~g}(8.51 \mathrm{mmol} ; 85 \%)$ of $\mathbf{1 b}$ as a white powder. ${ }^{1} \mathrm{H}$ NMR (400 $\mathrm{MHz}_{\mathrm{CDCl}}$ ): $\delta 1.90\left(\mathrm{~m}, 4 \mathrm{H}, \mathrm{CH}_{2} \mathrm{CH}_{2}\right), 3.44(\mathrm{t}, J=7.0 \mathrm{~Hz}, 2 \mathrm{H}$, $\left.\mathrm{NCH}_{2}\right), 3.61$ (t, $\left.J=7.0 \mathrm{~Hz}, 2 \mathrm{H}, \mathrm{NCH}_{2}\right) \cdot{ }^{13} \mathrm{C} \mathrm{NMR}(100 \mathrm{MHz}$, $\left.\mathrm{CDCl}_{3}\right): \delta 24.6\left(\mathrm{CH}_{2}\right), 25.3\left(\mathrm{CH}_{2}\right), 45.4\left(\mathrm{NCH}_{2}\right), 48.0\left(\mathrm{NCH}_{2}\right), 53.8$ $(\mathrm{C} \equiv C \mathrm{Br}), 74.6(C \equiv \mathrm{CBr}), 177.4\left(\mathrm{C}(\mathrm{O}) \mathrm{NC}_{4} \mathrm{H}_{8}\right)$. IR $\left(\mathrm{CH}_{2} \mathrm{Cl}_{2}\right)$ : $v(\mathrm{CCC}) 2196 \mathrm{~cm}^{-1}$. EI-MS (70 eV): $\mathrm{m} / \mathrm{z}$ (\%) $202(79)\left[\mathrm{M}^{+}\right], 146$ (22) $\left[\left(\mathrm{M}-\mathrm{C}_{4} \mathrm{H}_{8}\right)^{+}\right], 132(100)\left[\left(\mathrm{M}-\mathrm{NC}_{4} \mathrm{H}_{8}\right)^{+}\right], 122(29)[(\mathrm{M}-$ $\left.\mathrm{Br})^{+}\right], 99(100)\left[(\mathrm{M}-\mathrm{Br}-\mathrm{C} \equiv \mathrm{C})^{+}\right]$. Anal. Calcd for $\mathrm{C}_{7} \mathrm{H}_{8} \mathrm{BrNO}$ (202.05): C, 41.61; H, 3.99; N, 6.93. Found: C, 42.46; H, 4.37; N, 8.21 .

trans-Bromobis(triphenylphosphine)(3-dimethylamino-3-oxy-1propynyl)palladium(II) (2a). A suspension of $1.16 \mathrm{~g}(1 \mathrm{mmol})$ of $\left[\mathrm{Pd}\left(\mathrm{PPh}_{3}\right)_{4}\right]$ in $30 \mathrm{~mL}$ of $\mathrm{CH}_{2} \mathrm{Cl}_{2}$ was treated with $0.26 \mathrm{~g}(1.5 \mathrm{mmol})$ of $1 \mathbf{a}$ at ambient temperature. The mixture was stirred for $30 \mathrm{~min}$, upon which it becomes a clear yellow solution. Then, $100 \mathrm{~mL}$ of dry $\mathrm{Et}_{2} \mathrm{O}$ was added. The light yellow precipitate was filtered off and washed repeatedly with $\mathrm{Et}_{2} \mathrm{O}(3 \times 30 \mathrm{~mL})$ and pentane $(2 \times$ $50 \mathrm{~mL}$ ). Repeated crystallization of the crude product from $\mathrm{CH}_{2} \mathrm{Cl}_{2}$ / $\mathrm{Et}_{2} \mathrm{O}$ gave $0.69 \mathrm{~g}(0.85 \mathrm{mmol} ; 85 \%)$ of pure $\mathbf{2 a}$ as an off-white powder. ${ }^{1} \mathrm{H}$ NMR (400 MHz, $\left.\mathrm{CD}_{2} \mathrm{Cl}_{2}\right): \delta 2.19\left(\mathrm{~s}, 3 \mathrm{H}, \mathrm{NCH}_{3}\right), 2.49$ (s, 3H, $\left.\mathrm{NCH}_{3}\right), 7.38-7.47$ (m, 18H, ArH), 7.68-7.73 (m, 12H, ArH). ${ }^{13} \mathrm{C} \mathrm{NMR}\left(100 \mathrm{MHz}, \mathrm{CD}_{2} \mathrm{Cl}_{2}\right): \delta 33.2\left(\mathrm{NCH}_{3}\right), 37.6\left(\mathrm{NCH}_{3}\right)$, $104.1\left(\mathrm{t},{ }^{2} J_{\mathrm{PC}}=10.8 \mathrm{~Hz}, \mathrm{Pd}-C \equiv \mathrm{C}\right), 107.7(\mathrm{Pd}-\mathrm{C} \equiv \mathrm{C}), 128.4(\mathrm{t}$, $\left.{ }^{3} J_{\mathrm{PC}}=4.8 \mathrm{~Hz}, m-\mathrm{C}\right), 130.9(p-\mathrm{C}), 131.4\left(\mathrm{t},{ }^{1} J_{\mathrm{PC}}=25.0 \mathrm{~Hz}, i-\mathrm{C}\right)$, $135.3\left(\mathrm{t},{ }^{2} J_{\mathrm{PC}}=6.7 \mathrm{~Hz}, o-\mathrm{C}\right), 154.4\left(C(\mathrm{O}) \mathrm{NMe}_{2}\right) .{ }^{31} \mathrm{P} \mathrm{NMR}(162$ $\left.\mathrm{MHz}, \mathrm{CD}_{2} \mathrm{Cl}_{2}\right): \delta$ 24.7. IR $\left(\mathrm{CH}_{2} \mathrm{Cl}_{2}\right): v(\mathrm{C} \equiv \mathrm{C}) 2109 \mathrm{~cm}^{-1} ; v(\mathrm{CO})$ $1609 \mathrm{~cm}^{-1}$. UV-vis $\left(\mathrm{CH}_{2} \mathrm{Cl}_{2}\right): \lambda_{\max }(\mathrm{nm})(\log \epsilon) 240$ (4.489), 305 (4.314). FAB-MS: $m / z$, \%) $725(28)\left[(\mathrm{M}-\mathrm{Br})^{+}\right]$. Anal. Calcd for $\mathrm{C}_{41} \mathrm{H}_{36} \mathrm{BrNOP} 2 \mathrm{Pd} \cdot \mathrm{CH}_{2} \mathrm{Cl}_{2}$ (807.01): C, 56.56; H, 4.29; N, 1.57. Found: C, 57.09; H, 4.60; N, 1.55.

trans-Bromobis(triphenylphosphine)(3-N,N-tetramethyleneamino3-oxy-1-propynyl)palladium(II) (2b). A suspension of $1.16 \mathrm{~g}$ $(1 \mathrm{mmol})$ of $\left[\mathrm{Pd}\left(\mathrm{PPh}_{3}\right)_{4}\right]$ in $30 \mathrm{~mL}$ of dry $\mathrm{CH}_{2} \mathrm{Cl}_{2}$ was treated with $0.30 \mathrm{~g}$ ( $1.5 \mathrm{mmol})$ of $\mathbf{1 b}$ at ambient temperature. The mixture was stirred for a further $30 \mathrm{~min}$, upon which it became a clear yellow solution. The solvent was removed in vacuo, and the crude product was purified by column chromatography using petroleum ether/ $\mathrm{CH}_{2} \mathrm{Cl}_{2}$ /acetone mixtures. Yield: $0.71 \mathrm{~g}(0.85 \mathrm{mmol} ; 85 \%)$ of $\mathbf{2 b}$ as a pale yellow powder. ${ }^{1} \mathrm{H} \mathrm{NMR}\left(400 \mathrm{MHz}, \mathrm{CDCl}_{3}\right): \delta 1.37(\mathrm{~m}$, $\left.2 \mathrm{H}, \mathrm{CH}_{2}\right), 1.56\left(\mathrm{~m}, 2 \mathrm{H}, \mathrm{CH}_{2}\right), 2.26\left(\mathrm{t}, J=7.0 \mathrm{~Hz}, 2 \mathrm{H}, \mathrm{NCH}_{2}\right)$, $3.03\left(\mathrm{t}, J=7.0 \mathrm{~Hz}, 2 \mathrm{H}, \mathrm{NCH}_{2}\right), 7.34-7.40(\mathrm{~m}, 18 \mathrm{H}, \mathrm{ArH})$, 7.67-7.72 (m, $12 \mathrm{H}, \mathrm{ArH}) .{ }^{13} \mathrm{C}$ NMR $\left(100 \mathrm{MHz}, \mathrm{CDCl}_{3}\right): \delta 24.6$ $\left(\mathrm{CH}_{2}\right) 25.0\left(\mathrm{CH}_{2}\right), 44.2\left(\mathrm{NCH}_{2}\right), 46.9\left(\mathrm{NCH}_{2}\right), 105.0\left(\mathrm{t},{ }^{3} \mathrm{~J}_{\mathrm{PC}}=5.8\right.$ $\mathrm{Hz}, \mathrm{Pd}-\mathrm{C} \equiv C), 107.1\left(\mathrm{t},{ }^{2} J_{\mathrm{PC}}=13.4 \mathrm{~Hz}, \mathrm{Pd}-C \equiv \mathrm{C}\right), 128.0\left(\mathrm{t},{ }^{3} J_{\mathrm{PC}}\right.$ $=4.8 \mathrm{~Hz}, m-\mathrm{C}), 130.4(p-\mathrm{C}), 130.9\left(\mathrm{t},{ }^{1} J_{\mathrm{PC}}=24.9 \mathrm{~Hz}, i-\mathrm{C}\right), 135,0$ $\left(\mathrm{t},{ }^{2} J_{\mathrm{PC}}=6.7 \mathrm{~Hz}, o-\mathrm{C}\right), 152.9\left(C(\mathrm{O}) \mathrm{NC}_{4} \mathrm{H}_{8}\right) .{ }^{31} \mathrm{P} \mathrm{NMR}(162 \mathrm{MHz}$, $\left.\mathrm{CDCl}_{3}\right): \delta$ 21.7. IR $\left(\mathrm{CH}_{2} \mathrm{Cl}_{2}\right): v(\mathrm{CCC}) 2115 \mathrm{~cm}^{-1}$. UV-vis $\left(\mathrm{CH}_{2} \mathrm{Cl}_{2}\right): \lambda_{\max }(\mathrm{nm})(\log \epsilon) 302$ (4.373). FAB-MS: $m / z$ (\%) 834 (7) $\left[\mathrm{M}^{+}\right], 751(8)\left[(\mathrm{M}-\mathrm{Br}-2 \mathrm{H})^{+}\right], 489(16)[(\mathrm{M}-\mathrm{Br}-2 \mathrm{H}-$ $\left.\mathrm{PPh}_{3}\right)^{+}$], 367 (41) [(M $\left.\left.-\mathrm{Br}-2 \mathrm{H}-\mathrm{PPh}_{3}-\mathrm{C}_{7} \mathrm{H}_{8} \mathrm{NO}\right)^{+}\right]$. Anal. Calcd for $\mathrm{C}_{43} \mathrm{H}_{38} \mathrm{BrNOP}{ }_{2} \mathrm{Pd}$ (833.04): C, 62.00; H, 4.60; N, 1.68 . Found: C, 61.86; H, 4.63; N, 1.57 .

trans-Bromobis(triphenylphosphine)(3-dimethylamino-3-methoxy-1,2-propadienylidene)palladium(II) Trifluoromethanesulfonate (3a-OTf). A solution of $0.5 \mathrm{~g}(0.62 \mathrm{mmol})$ of $2 \mathrm{a}$ in $30 \mathrm{~mL}$ of $\mathrm{CH}_{2} \mathrm{Cl}_{2}$ was treated dropwise with $0.07 \mathrm{~mL}(0.62 \mathrm{mmol})$ of MeOTf at -50 
${ }^{\circ} \mathrm{C}$. After $10 \mathrm{~min}$ at $-50{ }^{\circ} \mathrm{C}$, the solution was warmed to ambient temperature. The progress of the reaction was followed by IR spectroscopy. When all of the starting material was consumed, the solvent was removed in vacuo. The remaining residue was washed twice with $30 \mathrm{~mL}$ of $\mathrm{Et}_{2} \mathrm{O}$ and recrystallized from mixtures of $\mathrm{CH}_{2} \mathrm{Cl}_{2}$ and pentane. Pure 3a-OTf (0.55 g, $0.56 \mathrm{mmol}$; 91\%) was obtained as a light yellow powder. ${ }^{1} \mathrm{H} \mathrm{NMR}\left(400 \mathrm{MHz}, \mathrm{CD}_{2} \mathrm{Cl}_{2}\right)$ : $\delta 3.23\left(\mathrm{~s}, 3 \mathrm{H}, \mathrm{NCH}_{3}\right), 3.40\left(\mathrm{~s}, 3 \mathrm{H}, \mathrm{NCH}_{3}\right), 3.74\left(\mathrm{~s}, 3 \mathrm{H}, \mathrm{OCH}_{3}\right)$, $7.03-7.11(\mathrm{~m}, 18 \mathrm{H}, \mathrm{ArH}), 7.21-7.25(\mathrm{~m}, 12 \mathrm{H}, \mathrm{ArH}) .{ }^{13} \mathrm{C} \mathrm{NMR}$ $\left(100 \mathrm{MHz}, \mathrm{CD}_{2} \mathrm{Cl}_{2}\right): \delta 35.1\left(\mathrm{NCH}_{3}\right), 40.3\left(\mathrm{NCH}_{3}\right), 61.7\left(\mathrm{OCH}_{3}\right)$, $96.1\left(\mathrm{C}_{\beta}\right), 120.9\left(\mathrm{q},{ }^{1} J_{\mathrm{CF}}=316.4 \mathrm{~Hz}, \mathrm{CF}_{3}\right), 128.9\left(\mathrm{t},{ }^{3} J_{\mathrm{PC}}=5.8\right.$ $\mathrm{Hz}, m-\mathrm{C}), 129.9\left(\mathrm{t},{ }^{1} J_{\mathrm{PC}}=25.3 \mathrm{~Hz}, i-\mathrm{C}\right), 132.2(p-\mathrm{C}), 135.4(\mathrm{t}$, $\left.{ }^{2} J_{\mathrm{PC}}=6.0 \mathrm{~Hz}, o-\mathrm{C}\right), 150.9\left(\mathrm{t},{ }^{3} J_{\mathrm{PC}}=5.5 \mathrm{~Hz}, \mathrm{C}_{\alpha}\right), 154.1\left(\mathrm{C}_{\gamma}\right) \cdot{ }^{31} \mathrm{P}$ NMR (162 MHz, $\mathrm{CD}_{2} \mathrm{Cl}_{2}$ ): $\delta$ 24.5. IR (THF): $v(\mathrm{CCC}) 2099 \mathrm{~cm}^{-1}$. $\mathrm{UV}-\mathrm{vis}\left(\mathrm{CH}_{2} \mathrm{Cl}_{2}\right): \lambda_{\max }, \mathrm{nm}(\log \epsilon) 236$ (4.563), 298 (4.361). FABMS: $m / z(\%) 821(56)\left[\left(\mathrm{M}-\mathrm{CF}_{3} \mathrm{SO}_{3}\right)^{+}\right], 560(48)\left[\left(\mathrm{M}-\mathrm{CF}_{3} \mathrm{SO}_{3}\right.\right.$ - $\left.\mathrm{PPh}_{3}\right)^{+}$]. Anal. Calcd for $\mathrm{C}_{43} \mathrm{H}_{39} \mathrm{BrF}_{3} \mathrm{NO}_{4} \mathrm{P}_{2} \mathrm{PdS}$ (971.11): C, 53.18; H, 4.05; N, 1.44. Found: C, 53.49; H, 4.46; N, 1.34 .

trans-Bromobis(triphenylphosphine)(3-dimethylamino-3-methoxy-1,2-propadienylidene)palladium(II) Tetrafluoroborate (3a$\left.\mathbf{B F}_{4}\right)$. The synthesis of $\mathbf{3 a}-\mathrm{BF}_{4}$ from $1.0 \mathrm{~g}(1.24 \mathrm{mmol})$ of $\mathbf{2 a}$ and $0.25 \mathrm{~g}$ (1.70 mmol, 1.4 equiv) of $\left[\mathrm{Me}_{3} \mathrm{O}\right] \mathrm{BF}_{4}$ in $60 \mathrm{~mL}$ of $\mathrm{CH}_{2} \mathrm{Cl}_{2}$ was carried out analogously to 3a-OTf. Yield: $0.70 \mathrm{~g}(0.77 \mathrm{mmol}$; $62 \%$ ) of $3 \mathbf{a}-\mathrm{BF}_{4}$ as a yellow powder. ${ }^{1} \mathrm{H}$ NMR (400 $\left.\mathrm{MHz}, \mathrm{CD}_{2} \mathrm{Cl}_{2}\right)$ : $\delta 2.69\left(\mathrm{~s}, 3 \mathrm{H}, \mathrm{NCH}_{3}\right), 2.91\left(\mathrm{~s}, 3 \mathrm{H}, \mathrm{NCH}_{3}\right), 3.31\left(\mathrm{~s}, 3 \mathrm{H}, \mathrm{OCH}_{3}\right)$, 7.45-7.56 (m, $18 \mathrm{H}, \mathrm{ArH}), 7.66-7.71(\mathrm{~m}, 12 \mathrm{H}, \mathrm{ArH}) .{ }^{13} \mathrm{C} \mathrm{NMR}$ $\left(100 \mathrm{MHz}, \mathrm{CD}_{2} \mathrm{Cl}_{2}\right): \delta 37.7\left(\mathrm{NCH}_{3}\right), 41.7\left(\mathrm{NCH}_{3}\right), 61.3\left(\mathrm{OCH}_{3}\right)$, $128.8\left(\mathrm{t},{ }^{3} J_{\mathrm{PC}}=5.6 \mathrm{~Hz}, m-\mathrm{C}\right), 130.2\left(\mathrm{t},{ }^{1} J_{\mathrm{PC}}=25.7 \mathrm{~Hz}, i-\mathrm{C}\right), 131.8$ $(p-\mathrm{C}), 135.1\left(\mathrm{t},{ }^{2} J_{\mathrm{PC}}=6.4 \mathrm{~Hz}, o-\mathrm{C}\right) ; \mathrm{C}_{\alpha}, \mathrm{C}_{\beta}, \mathrm{C}_{\gamma}$ not observed. ${ }^{31} \mathrm{P}$ NMR (162 MHz, $\left.\mathrm{CD}_{2} \mathrm{Cl}_{2}\right): \delta$ 24.7. IR $\left(\mathrm{CH}_{2} \mathrm{Cl}_{2}\right): v(\mathrm{CCC}) 2098 \mathrm{~cm}^{-1}$. $\mathrm{UV}-$ vis $\left(\mathrm{CH}_{2} \mathrm{Cl}_{2}\right): \lambda_{\max }(\mathrm{nm})(\log \epsilon) 298$ (4.368). FAB-MS: $\mathrm{m} / \mathrm{z}$ (\%) $822(72)\left[\left(\mathrm{M}-\mathrm{BF}_{4}\right)^{+}\right], 560(37)\left[\left(\mathrm{M}-\mathrm{BF}_{4}-\mathrm{PPh}_{3}\right)^{+}\right]$. Anal. Calcd for $\mathrm{C}_{42} \mathrm{H}_{39} \mathrm{BBrF}_{4} \mathrm{NOP}_{2} \mathrm{Pd} \cdot 0.5 \mathrm{CH}_{2} \mathrm{Cl}_{2}$ (908.85): C, 53.66; $\mathrm{H}$, 4.24; N, 1.47. Found: C, 53.83; H, 4.51; N, 1.49.

trans-Bromobis(triphenylphosphine)(3- $N, N$-tetramethyleneamino3-methoxy-1,2-propadienylidene)palladium(II) Trifluoromethanesulfonate (3b-OTf). The synthesis of 3b-OTf from $0.32 \mathrm{~g}(0.38$ $\mathrm{mmol})$ of $\mathbf{2} \mathbf{b}$ and $0.04 \mathrm{~mL}(0.38 \mathrm{mmol})$ of MeOTf in $20 \mathrm{~mL}$ of $\mathrm{CH}_{2} \mathrm{Cl}_{2}$ was carried out analogously to 3a-OTf. Yield: $0.82 \mathrm{~g}(0.82$ mmol; 97\%) of 3b-OTf as a yellow powder. ${ }^{1} \mathrm{H}$ NMR $(400 \mathrm{MHz}$, $\left.\mathrm{CD}_{2} \mathrm{Cl}_{2}\right): \delta 1.70\left(\mathrm{~m}, 2 \mathrm{H}, \mathrm{CH}_{2}\right), 1.87\left(\mathrm{~m}, 2 \mathrm{H}, \mathrm{CH}_{2}\right), 2.68(\mathrm{t}, J=$ $\left.7.0 \mathrm{~Hz}, 2 \mathrm{H}, \mathrm{NCH}_{2}\right), 3.27\left(\mathrm{~s}, 3 \mathrm{H}, \mathrm{OCH}_{3}\right), 3.34(\mathrm{t}, J=7.0 \mathrm{~Hz}, 2$ $\left.\mathrm{H}, \mathrm{NCH}_{2}\right), 7.42-7.50$ (m, $\left.18 \mathrm{H}, \mathrm{ArH}\right), 7.63-7.67$ (m, $\left.12 \mathrm{H}, \mathrm{ArH}\right)$. ${ }^{13} \mathrm{C}$ NMR $\left(100 \mathrm{MHz}, \mathrm{CD}_{2} \mathrm{Cl}_{2}\right): \delta 24.2\left(\mathrm{CH}_{2}\right), 24.4\left(\mathrm{CH}_{2}\right), 48.9$ $\left(\mathrm{NCH}_{2}\right), 51.4\left(\mathrm{NCH}_{2}\right), 60.4\left(\mathrm{OCH}_{3}\right), 93.8\left(\mathrm{C}_{\beta}\right), 128.5\left(\mathrm{t},{ }^{3} J_{\mathrm{PC}}=\right.$ $5.6 \mathrm{~Hz}, m-\mathrm{C}), 129.8\left(\mathrm{t},{ }^{1} J_{\mathrm{PC}}=25.9 \mathrm{~Hz}, i-\mathrm{C}\right), 131.4(p-\mathrm{C}), 134.7(\mathrm{t}$, $\left.{ }^{2} J_{\mathrm{PC}}=6.2 \mathrm{~Hz}, o-\mathrm{C}\right), 147.8\left(\mathrm{C}_{\alpha}\right), 150.9\left(\mathrm{C}_{\gamma}\right) .{ }^{31} \mathrm{P} \mathrm{NMR}(162 \mathrm{MHz}$, $\left.\mathrm{CDCl}_{3}\right): \delta$ 24.9. IR $\left(\mathrm{CH}_{2} \mathrm{Cl}_{2}\right): v(\mathrm{CCC}) 2100 \mathrm{~cm}^{-1} ; v(\mathrm{CO}) 1612$ $\mathrm{cm}^{-1}$. UV-vis $\left(\mathrm{CH}_{2} \mathrm{Cl}_{2}\right): \lambda_{\max }(\mathrm{nm})(\log \epsilon) 296$ (4.437). FAB-MS: $\mathrm{m} / \mathrm{z}(\%) 847(32)\left[(\mathrm{M}-\mathrm{OTf})^{+}\right], 586(20)\left[\left(\mathrm{M}-\mathrm{OTf}-\mathrm{PPh}_{3}\right)^{+}\right]$, 505 (11) $\left[\left(\mathrm{M}-\mathrm{OTf}-\mathrm{PPh}_{3}-\mathrm{Br}\right)^{+}\right], 323(59)[(\mathrm{M}-\mathrm{OTf}-2$ $\left.\mathrm{PPh}_{3}\right)^{+}$]. Anal. Calcd for $\mathrm{C}_{45} \mathrm{H}_{41} \mathrm{BrF}_{3} \mathrm{NO}_{4} \mathrm{P}_{2} \mathrm{PdS}$ (997.15): C, 54.20; H, 4.14; N, 1.40. Found: C, 54.16; H, 4.19; N, 1.36 .

\footnotetext{
trans-Bromobis(triphenylphosphine)(3-N,N-tetramethyleneamino3-methoxy-1,2-propadienylidene)palladium(II) Tetrafluoroborate (3b-BF 4 ). The synthesis of $\mathbf{3 b}-\mathrm{BF}_{4}$ from $0.48 \mathrm{~g}(0.58 \mathrm{mmol})$ of $\mathbf{2 b}$ and $0.10 \mathrm{~g}$ (0.69 mmol, 1.2 equiv) of $\left[\mathrm{Me}_{3} \mathrm{O}\right] \mathrm{BF}_{4}$ in $30 \mathrm{~mL}$ of $\mathrm{CH}_{2} \mathrm{Cl}_{2}$ was carried out analogously to 3a-OTf. Yield: $0.52 \mathrm{~g}(0.56$ mmol; 97\%) of $\mathbf{3 b}-\mathrm{BF}_{4}$ as a yellow powder. ${ }^{1} \mathrm{H}$ NMR $(400 \mathrm{MHz}$, $\left.\mathrm{CDCl}_{3}\right): \delta 1.69\left(\mathrm{~m}, 2 \mathrm{H}, \mathrm{CH}_{2}\right), 1.86\left(\mathrm{~m}, 2 \mathrm{H}, \mathrm{CH}_{2}\right), 2.67(\mathrm{t}, J=7.0$ $\left.\mathrm{Hz}, 2 \mathrm{H}, \mathrm{NCH}_{2}\right), 3.24\left(\mathrm{~s}, 3 \mathrm{H}, \mathrm{OCH}_{3}\right), 3.34(\mathrm{t}, J=7.0 \mathrm{~Hz}, 2 \mathrm{H}$, $\mathrm{NCH}_{2}$ ), 7.37-7.51 (m, $\left.18 \mathrm{H}, \mathrm{ArH}\right), 7.63-7.70$ (m, $\left.12 \mathrm{H}, \mathrm{ArH}\right)$. ${ }^{13} \mathrm{C}$ NMR $\left(100 \mathrm{MHz}, \mathrm{CDCl}_{3}\right): \delta 24.5\left(\mathrm{CH}_{2}\right), 24.7\left(\mathrm{CH}_{2}\right), 49.2$ $\left(\mathrm{NCH}_{2}\right), 51.7\left(\mathrm{NCH}_{2}\right), 60.7\left(\mathrm{OCH}_{3}\right), 94.3\left(\mathrm{C}_{\beta}\right), 128.8\left(\mathrm{t},{ }^{3} J_{\mathrm{PC}}=\right.$ $5.3 \mathrm{~Hz}, m-\mathrm{C}), 130.1\left(\mathrm{t},{ }^{1} J_{\mathrm{PC}}=26.0 \mathrm{~Hz}, i-\mathrm{C}\right), 131.7(p-\mathrm{C}), 135.1(\mathrm{t}$, $\left.{ }^{2} J_{\mathrm{PC}}=6.2 \mathrm{~Hz}, o-\mathrm{C}\right), 151.2\left(\mathrm{C}_{\alpha}\right), 154.1\left(\mathrm{C}_{\gamma}\right) .{ }^{31} \mathrm{P} \mathrm{NMR}(162 \mathrm{MHz}$, $\left.\mathrm{CD}_{2} \mathrm{Cl}_{2}\right): \delta$ 25.0. IR $\left(\mathrm{CH}_{2} \mathrm{Cl}_{2}\right): v(\mathrm{CCC}) 2101 \mathrm{~cm}^{-1} ; v(\mathrm{CO}) 1609$
}

$\mathrm{cm}^{-1}$. UV-vis $\left(\mathrm{CH}_{2} \mathrm{Cl}_{2}\right): \lambda_{\max }(\mathrm{nm})(\log \epsilon) 300$ (4.376). FAB-MS: $\mathrm{m} / \mathrm{z}(\%) 847(19)\left[\left(\mathrm{M}-\mathrm{BF}_{4}\right)^{+}\right], 586(16)\left[\left(\mathrm{M}-\mathrm{BF}_{4}-\mathrm{PPh}_{3}\right)^{+}\right]$, 505 (9) $\left[\left(\mathrm{M}-\mathrm{BF}_{4}-\mathrm{PPh}_{3}-\mathrm{Br}\right)^{+}\right], 324$ (40) $\left[\left(\mathrm{M}-\mathrm{BF}_{4}-2\right.\right.$ $\left.\mathrm{PPh}_{3}\right)^{+}$]. Anal. Calcd for $\mathrm{C}_{44} \mathrm{H}_{41} \mathrm{BBrF}_{4} \mathrm{NOP}_{2} \mathrm{Pd} \cdot 0.5 \mathrm{CH}_{2} \mathrm{Cl}_{2}$ (934.89): C, 54.69; H, 4.33; N, 1.43. Found: C, 54.52; H, 4.51; N, 1.35 .

trans-Bromobis(triphenylphosphine)(3-dimethylamino-3-ethoxy1,2-propadienylidene)palladium(II) Tetrafluoroborate $\left(4 \mathrm{a}-\mathrm{BF}_{4}\right)$. The synthesis of $\mathbf{4 a}-\mathrm{BF}_{4}$ from $91 \mathrm{mg}(0.11 \mathrm{mmol})$ of $\mathbf{2 a}$ and 21 $\mathrm{mg}(0.11 \mathrm{mmol})$ of $\left[\mathrm{Et}_{3} \mathrm{O}\right] \mathrm{BF}_{4}$ in $5 \mathrm{~mL}$ of $\mathrm{CH}_{2} \mathrm{Cl}_{2}$ was carried out analogously to 3a-OTf. Yield: $77 \mathrm{mg}(0.08 \mathrm{mmol}$; 76\%) of 4a$\mathrm{BF}_{4}$ as a yellow powder. ${ }^{1} \mathrm{H} \mathrm{NMR}\left(400 \mathrm{MHz}, \mathrm{CD}_{2} \mathrm{Cl}_{2}\right): \delta 1.01(\mathrm{t}$, $\left.J=7.0 \mathrm{~Hz}, 2 \mathrm{H}, \mathrm{CH}_{3}\right), 2.69$ (s, $\left.3 \mathrm{H}, \mathrm{NCH}_{3}\right), 2.90\left(\mathrm{~s}, 3 \mathrm{H}, \mathrm{NCH}_{3}\right)$, $3.54\left(\mathrm{q}, J=7.0 \mathrm{~Hz}, 2 \mathrm{H}, \mathrm{CH}_{2}\right), 7.45-7.56(\mathrm{~m}, 18 \mathrm{H}, \mathrm{ArH})$, 7.67-7.72 (m, $\left.12 \mathrm{H}, \mathrm{ArH}) .{ }^{13} \mathrm{C} \mathrm{NMR} \mathrm{(100} \mathrm{MHz,} \mathrm{CD}_{2} \mathrm{Cl}_{2}\right): \delta 14.3$ $\left(\mathrm{OCH}_{2} \mathrm{CH}_{3}\right), 37.5\left(\mathrm{NCH}_{3}\right), 41.4\left(\mathrm{NCH}_{3}\right), 71.9\left(\mathrm{OCH}_{2} \mathrm{CH}_{3}\right), 93.1$ $\left(\mathrm{t},{ }^{3} J_{\mathrm{PC}}=4.8 \mathrm{~Hz}, \mathrm{C}_{\beta}\right), 128.8\left(\mathrm{t},{ }^{3} J_{\mathrm{PC}}=5.8 \mathrm{~Hz}, m-\mathrm{C}\right), 130.1\left(\mathrm{t},{ }^{1} J_{\mathrm{PC}}\right.$ $=25.9 \mathrm{~Hz}, i-\mathrm{C}), 131.7(p-\mathrm{C}), 135.0\left(\mathrm{t},{ }^{2} J_{\mathrm{PC}}=5.8 \mathrm{~Hz}, o-\mathrm{C}\right), 149.3$ $\left(\mathrm{t},{ }^{2} J_{\mathrm{PC}}=12.5 \mathrm{~Hz}, \mathrm{C}_{\alpha}\right), 152.9\left(\mathrm{C}_{\gamma}\right) .{ }^{31} \mathrm{P}$ NMR $\left(162 \mathrm{MHz}, \mathrm{CD}_{2} \mathrm{Cl}_{2}\right)$ : $\delta$ 24.8. IR $\left(\mathrm{CH}_{2} \mathrm{Cl}_{2}\right): v(\mathrm{CCC}) 2099 \mathrm{~cm}^{-1}$. UV-vis $\left(\mathrm{CH}_{2} \mathrm{Cl}_{2}\right): \lambda_{\max }$ (nm) $(\log \epsilon) 300$ (4.335). FAB-MS: $m / z(\%) 834(44)\left[\left(\mathrm{M}-\mathrm{BF}_{4}\right)^{+}\right]$, 573 (18) $\left[\left(\mathrm{M}-\mathrm{BF}_{4}-\mathrm{PPh}_{3}\right)^{+}\right]$. Anal. Calcd for $\mathrm{C}_{43} \mathrm{H}_{41} \mathrm{BBrF}_{4} \mathrm{NOP}_{2} \mathrm{Pd}$ (922.88): C, 55.96; $\mathrm{H}, 4.48 ; \mathrm{N}, 1.52$. Found: C, 56.02; H, 4.50; N, 1.44 .

trans-Bromobis(triphenylphosphine)(3-N,N-tetramethyleneamino3-ethoxy-1,2-propadienylidene)palladium(II) Tetrafluoroborate (4b$\left.\mathbf{B F}_{\mathbf{4}}\right)$. The synthesis of $\mathbf{4 b}-\mathrm{BF}_{4}$ from $0.81 \mathrm{~g}(0.97 \mathrm{mmol})$ of $\mathbf{2} \mathbf{b}$ and $0.18 \mathrm{~g}(0.97 \mathrm{mmol})$ of $\left[\mathrm{Et}_{3} \mathrm{O}\right] \mathrm{BF}_{4}$ in $30 \mathrm{~mL}$ of $\mathrm{CH}_{2} \mathrm{Cl}_{2}$ was carried out analogously to 3a-OTf. Yield: $0.92 \mathrm{~g}$ (0.96 mmol; 99\%) of 4b- $\mathrm{BF}_{4}$ as a yellow powder. ${ }^{1} \mathrm{H} \mathrm{NMR}\left(400 \mathrm{MHz}, \mathrm{CD}_{2} \mathrm{Cl}_{2}\right): \delta 0.95$ (t, $\left.J=7.0 \mathrm{~Hz}, 3 \mathrm{H}, \mathrm{OCH}_{2} \mathrm{CH}_{3}\right), 1.86\left(\mathrm{~m}, 2 \mathrm{H}, \mathrm{CH}_{2}\right), 2.67$ (t, $J=$ $\left.7.0 \mathrm{~Hz}, 2 \mathrm{H}, \mathrm{NCH}_{2}\right), 3.31\left(\mathrm{t}, J=7.0 \mathrm{~Hz}, 2 \mathrm{H}, \mathrm{NCH}_{2}\right), 3.48$ (q, $J$ $\left.=7.0 \mathrm{~Hz}, 2 \mathrm{H}, \mathrm{OCH}_{2} \mathrm{CH}_{3}\right), 7.42-7.50(\mathrm{~m}, 18 \mathrm{H}, \mathrm{ArH}), 7.62-7.67$ (m, $12 \mathrm{H}, \mathrm{ArH}) .{ }^{13} \mathrm{C}$ NMR (100 MHz, $\left.\mathrm{CD}_{2} \mathrm{Cl}_{2}\right): \delta 14.2\left(\mathrm{OCH}_{2} \mathrm{CH}_{3}\right)$, $24.1\left(\mathrm{CH}_{2}\right), 24.3\left(\mathrm{CH}_{2}\right), 48.8\left(\mathrm{NCH}_{2}\right), 51.3\left(\mathrm{NCH}_{2}\right), 70.7$ $\left(\mathrm{OCH}_{2} \mathrm{CH}_{3}\right), 94.1\left(\mathrm{C}_{\beta}\right), 128.4\left(\mathrm{t},{ }^{3} J_{\mathrm{PC}}=5.7 \mathrm{~Hz}, m-\mathrm{C}\right), 129.8(\mathrm{t}$, $\left.{ }^{1} J_{\mathrm{PC}}=26.0 \mathrm{~Hz}, i-\mathrm{C}\right), 131.4(p-\mathrm{C}), 134.7\left(\mathrm{t},{ }^{2} J_{\mathrm{PC}}=5.7 \mathrm{~Hz}, o-\mathrm{C}\right)$, $145.8\left(\mathrm{C}_{\alpha}\right), 150.1(\mathrm{C} \gamma) \cdot{ }^{31} \mathrm{P}$ NMR $\left(162 \mathrm{MHz}, \mathrm{CD}_{2} \mathrm{Cl}_{2}\right): \delta 21.8$. IR $\left(\mathrm{CH}_{2} \mathrm{Cl}_{2}\right): v(\mathrm{CCC}) 2101 \mathrm{~cm}^{-1} ; \quad v(\mathrm{CO}) 1604 \mathrm{~cm}^{-1}$. UV-vis $\left(\mathrm{CH}_{2} \mathrm{Cl}_{2}\right): \lambda_{\max }(\mathrm{nm})(\log \epsilon) 297$ (4.462). FAB-MS: $m / z$ (\%) 861 (50) $\left[\left(\mathrm{M}-\mathrm{BF}_{4}\right)^{+}\right], 600(37)\left[\left(\mathrm{M}-\mathrm{BF}_{4}-\mathrm{PPh}_{3}\right)^{+}\right]$. Anal. Calcd for $\mathrm{C}_{45} \mathrm{H}_{43} \mathrm{BBrF}_{4} \mathrm{NOP}_{2} \mathrm{Pd}$ (948.92): C, 56.96; H, 4.57; N, 1.48 . Found: C, 56.83; H, 4.53; N, 1.55 .

trans-Bromobis(triisopropylphosphine)(3-dimethylamino-3-oxy1-propynyl)palladium(II) (5a). At ambient temperature, a solution of $0.55 \mathrm{~g}(0.68 \mathrm{mmol})$ of $2 \mathrm{a}$ in $30 \mathrm{~mL}$ of $\mathrm{CH}_{2} \mathrm{Cl}_{2}$ was treated with $0.29 \mathrm{~mL}$ (1.50 mmol, 2.2 equiv) of $\mathrm{P}^{i} \mathrm{Pr}_{3}$. The progress of the reaction was monitored by IR spectroscopy. When all of the starting material was consumed (60 $\mathrm{min})$, the solvent was removed in vacuo and the crude product purified by column chromatography using a petroleum ether/ $/ \mathrm{Et}_{2} \mathrm{O}$ mixture as the eluant. Removal of the solvent gave $0.29 \mathrm{~g}(0.47 \mathrm{mmol}, 70 \%)$ of pure $\mathbf{5 a}$ as a white powder. ${ }^{1} \mathrm{H}$ NMR (400 MHz, $\mathrm{CD}_{2} \mathrm{Cl}_{2}$ ): $\delta 1.35$ (q, $\left.J=7.04 \mathrm{~Hz}, 36 \mathrm{H}, \mathrm{CH}\left(\mathrm{CH}_{3}\right)_{2}\right)$, $2.82\left(\mathrm{~s}, 3 \mathrm{H}, \mathrm{NCH}_{3}\right), 2.89\left(\mathrm{~m}, 6 \mathrm{H}, \mathrm{CH}\left(\mathrm{CH}_{3}\right)_{2}\right), 3.10\left(\mathrm{~s}, 3 \mathrm{H}, \mathrm{NCH}_{3}\right)$. ${ }^{13} \mathrm{C} \mathrm{NMR}\left(100 \mathrm{MHz}, \mathrm{CD}_{2} \mathrm{Cl}_{2}\right): \delta 20.1\left(\mathrm{CH}\left(\mathrm{CH}_{3}\right)_{2}\right), 24.7\left(\mathrm{t},{ }^{2} \mathrm{~J}_{\mathrm{PC}}=\right.$ $\left.11.5 \mathrm{~Hz}, \mathrm{CH}\left(\mathrm{CH}_{3}\right)_{2}\right), 33.7\left(\mathrm{NCH}_{3}\right), 38.0\left(\mathrm{NCH}_{3}\right), 103.7\left(\mathrm{t},{ }^{3} J_{\mathrm{PC}}=\right.$ $4.8 \mathrm{~Hz}, \mathrm{Pd}-\mathrm{C} \equiv C), 107.7\left(\mathrm{t},{ }^{2} J_{\mathrm{PC}}=12.6 \mathrm{~Hz}, \mathrm{Pd}-C \equiv \mathrm{C}\right), 155.3$ $\left(\mathrm{C}(\mathrm{O}) \mathrm{NMe}_{2}\right) .{ }^{31} \mathrm{P}$ NMR (162 MHz, $\left.\mathrm{CD}_{2} \mathrm{Cl}_{2}\right): \delta$ 42.1. IR (THF): $v(\mathrm{C} \equiv \mathrm{C}) 2098 \mathrm{~cm}^{-1} ; v(\mathrm{CO}) 1605 \mathrm{~cm}^{-1}$. UV-vis $\left(\mathrm{CH}_{2} \mathrm{Cl}_{2}\right): \lambda_{\max }$ (nm) $(\log \epsilon) 251$ (4.134), 288 (4.167). MS (FAB): $m / z$ (\%) 604 (19) $\left[\mathrm{M}^{+}\right], 523(24)\left[(\mathrm{M}-\mathrm{Br})^{+}\right], 362(11)\left[\left(\mathrm{M}-\mathrm{Br}-\mathrm{P}\left({ }^{i} \mathrm{Pr}\right)_{3}\right)^{+}\right]$. Anal. Calcd for $\mathrm{C}_{23} \mathrm{H}_{48} \mathrm{BrNOP}{ }_{2} \mathrm{Pd}$ (602.91): C, 45.82; H, 8.02; N, 2.32. Found: C, 46.03; H, 7.97; N, 2.39 .

trans-Bromobis(triisopropylphosphine)(3-N,N-tetramethyleneamino-3-oxy-1-propynyl)palladium(II) (5b). The synthesis of $\mathbf{5 b}$ from $1.23 \mathrm{~g}$ ( $1.48 \mathrm{mmol})$ of $\mathbf{2 b}$ and $0.62 \mathrm{~mL}$ (3.25 mmol, 2.2 equiv) of $\mathrm{P}^{i} \mathrm{Pr}_{3}$ in $30 \mathrm{~mL}$ of $\mathrm{CH}_{2} \mathrm{Cl}_{2}$ was carried out analogously to $5 \mathbf{a}$. The crude product was purified by column chromatography using 
an ether/ $\mathrm{CH}_{2} \mathrm{Cl}_{2}$ /acetone mixture. Yield: $0.65 \mathrm{~g}(1.03 \mathrm{mmol} ; 70 \%)$ of $\mathbf{5 b}$ as a white powder. ${ }^{1} \mathrm{H} \mathrm{NMR}\left(400 \mathrm{MHz}, \mathrm{CDCl}_{3}\right): \delta 1.38(\mathrm{~m}$, $\left.36 \mathrm{H}, \mathrm{CH}\left(\mathrm{CH}_{3}\right)_{2}\right), 1.87$ (br, $\left.4 \mathrm{H}, \mathrm{CH}_{2} \mathrm{CH}_{2}\right), 2.94\left(\mathrm{~m}, 6 \mathrm{H}, \mathrm{CH}\left(\mathrm{CH}_{3}\right)_{2}\right)$, $3.40\left(\mathrm{t}, J=6.6 \mathrm{~Hz}, 2 \mathrm{H}, \mathrm{NCH}_{2}\right), 3.53\left(\mathrm{t}, J=6.6 \mathrm{~Hz}, 2 \mathrm{H}, \mathrm{NCH}_{2}\right)$. ${ }^{13} \mathrm{C} \mathrm{NMR}\left(100 \mathrm{MHz}, \mathrm{CDCl}_{3}\right): \delta 19.6\left(\mathrm{CH}\left(\mathrm{CH}_{3}\right)_{2}\right), 24.0\left(\mathrm{t},{ }^{2} J_{\mathrm{PC}}=\right.$ $\left.11.6 \mathrm{~Hz}, \mathrm{CH}\left(\mathrm{CH}_{3}\right)_{2}\right), 24.3\left(\mathrm{CH}_{2}\right), 25.2\left(\mathrm{CH}_{2}\right), 44.3\left(\mathrm{NCH}_{2}\right), 47.0$ $\left(\mathrm{NCH}_{2}\right), 103.9\left(\mathrm{t},{ }^{3} J_{\mathrm{PC}}=4.8 \mathrm{~Hz}, \mathrm{Pd}-\mathrm{C} \equiv \mathrm{C}\right), 106.0\left(\mathrm{t},{ }^{2} J_{\mathrm{PC}}=12.4\right.$ $\mathrm{Hz}, \mathrm{Pd}-C \equiv \mathrm{C}), 153.1\left(\mathrm{C}(\mathrm{O}) \mathrm{NC}_{4} \mathrm{H}_{8}\right) .{ }^{31} \mathrm{P} \mathrm{NMR}\left(162 \mathrm{MHz}, \mathrm{CDCl}_{3}\right)$ : $\delta$ 41.7. IR $\left(\mathrm{CH}_{2} \mathrm{Cl}_{2}\right): v(\mathrm{CCC}) 2106 \mathrm{~cm}^{-1}$. UV-vis $\left(\mathrm{CH}_{2} \mathrm{Cl}_{2}\right): \lambda_{\max }$ (nm) $(\log \epsilon) 287$ (4.224). FAB-MS: $m / z$ (\%) 629 (39) $\left[\mathrm{M}^{+}\right], 549$ (18) $\left[(\mathrm{M}-\mathrm{Br})^{+}\right], 470(6)\left[\left(\mathrm{M}-\mathrm{P}\left({ }^{i} \mathrm{Pr}\right)_{3}\right)^{+}\right], 389$ (9) $[(\mathrm{M}-\mathrm{Br}-$ $\left.\left.\mathrm{P}\left({ }^{i} \mathrm{Pr}\right)_{3}\right)^{+}\right], 347(6)\left[\left(\mathrm{M}-\mathrm{P}\left({ }^{i} \mathrm{Pr}\right)_{3}\right)^{+}-\mathrm{C}_{7} \mathrm{H}_{8} \mathrm{NO}\right], 267$ (38) [( $\mathrm{M}-$ $\left.\left.\mathrm{Br}-\mathrm{P}\left({ }^{i} \mathrm{Pr}\right)_{3}-\mathrm{C}_{7} \mathrm{H}_{8} \mathrm{NO}\right)^{+}\right]$. Anal. Calcd for $\mathrm{C}_{25} \mathrm{H}_{50} \mathrm{BrNOP}_{2} \mathrm{Pd}$ (628.95): C, 47.74; H, 8.01; N, 2,23. Found: C, 47.68; H, 7.74; N, 2.57 .

trans-Bromobis(triisopropylphosphine)(3-dimethylamino-3-methoxy-1,2-propadienylidene)palladium(II) Trifluoromethanesulfonate (6a-OTf). The synthesis of $\mathbf{6 a - O T f}$ from $0.3 \mathrm{~g}(0.50 \mathrm{mmol})$ of $\mathbf{5 a}$ and $0.06 \mathrm{~mL}(0.50 \mathrm{mmol})$ of MeOTf in $30 \mathrm{~mL}$ of $\mathrm{CH}_{2} \mathrm{Cl}_{2}$ was carried out analogously to 3a-OTf. Complex 6a-OTf was recrystallized from $\mathrm{Et}_{2} \mathrm{O}$. Yield: $0.37 \mathrm{~g}(0.49 \mathrm{mmol} ; 98 \%)$ of $\mathbf{6 a - O T f}$ as a colorless powder. ${ }^{1} \mathrm{H}$ NMR $\left(400 \mathrm{MHz}, \mathrm{CD}_{2} \mathrm{Cl}_{2}\right): \delta 1.35$ (q, $J=$ $\left.7.04 \mathrm{~Hz}, 36 \mathrm{H}, \mathrm{CH}\left(\mathrm{CH}_{3}\right)_{2}\right), 2.85\left(\mathrm{~m}, 6 \mathrm{H}, \mathrm{CH}\left(\mathrm{CH}_{3}\right)_{2}\right), 3.25(\mathrm{~s}, 3 \mathrm{H}$, $\left.\mathrm{NCH}_{3}\right), 3.45\left(\mathrm{~s}, 3 \mathrm{H}, \mathrm{NCH}_{3}\right), 4.20\left(\mathrm{~s}, 3 \mathrm{H}, \mathrm{OCH}_{3}\right) .{ }^{13} \mathrm{C} \mathrm{NMR}(100$ $\left.\mathrm{MHz}, \mathrm{CD}_{2} \mathrm{Cl}_{2}\right): \delta 20.0\left(\mathrm{CH}\left(\mathrm{CH}_{3}\right)_{2}\right), 25.0\left(\mathrm{t},{ }^{2} \mathrm{~J}_{\mathrm{PC}}=11.8 \mathrm{~Hz}\right.$, $\left.\mathrm{CH}\left(\mathrm{CH}_{3}\right)_{2}\right), 38.3\left(\mathrm{NCH}_{3}\right), 42.1\left(\mathrm{NCH}_{3}\right), 61.7\left(\mathrm{OCH}_{3}\right), 94.6\left(\mathrm{t},{ }^{3} J_{\mathrm{PC}}\right.$ $\left.=3.8 \mathrm{~Hz}, \mathrm{C}_{\beta}\right), 121.4\left(\mathrm{q},{ }^{1} J_{\mathrm{CF}}=320.0 \mathrm{~Hz}, \mathrm{CF}_{3}\right), 150.3\left(\mathrm{t},{ }^{2} J_{\mathrm{PC}}=\right.$ $\left.10.6 \mathrm{~Hz}, \mathrm{C}_{\alpha}\right), 153.7\left(\mathrm{C}_{\gamma}\right) .{ }^{31} \mathrm{P}$ NMR $\left(162 \mathrm{MHz}, \mathrm{CD}_{2} \mathrm{Cl}_{2}\right): \delta 45.3$. IR (THF): $v$ (CCC) $2083 \mathrm{~cm}^{-1}$. UV-vis $\left(\mathrm{CH}_{2} \mathrm{Cl}_{2}\right): \lambda_{\max }(\mathrm{nm})(\log$ є) 247 (4.103), 279 (4.371). FAB-MS: $m / z$ (\%) 618 (75) [(M $\left.\mathrm{OTf}^{+}\right], 458$ (48) $\left[\left(\mathrm{M}-\mathrm{OTf}-\mathrm{P}\left({ }^{i} \mathrm{Pr}\right)_{3}\right)^{+}\right]$. Anal. Calcd for $\mathrm{C}_{25} \mathrm{H}_{51} \mathrm{BrF}_{3} \mathrm{NO}_{4} \mathrm{P}_{2} \mathrm{PdS}$ (767.01): C, 40.80; H, 6.98; N, 1.90. Found: C, 39.38; H, 6.33; N, 1.54 .

trans-Bromobis(triisopropylphosphine)(3-N,N-tetramethyleneamino-3-methoxy-1,2-propadienylidene)palladium(II) Trifluoromethanesulfonate (6b-OTf). The synthesis of $\mathbf{6 b}-\mathrm{OTf}$ from $0.18 \mathrm{~g}$ $(0.27 \mathrm{mmol})$ of $\mathbf{5 b}$ and $0.03 \mathrm{~mL}(0.27 \mathrm{mmol})$ of MeOTf in $10 \mathrm{~mL}$ of $\mathrm{CH}_{2} \mathrm{Cl}_{2}$ was carried out analogously to 3a-OTf. Yield: $0.21 \mathrm{~g}$ $(0.26 \mathrm{mmol} ; 97 \%)$ of $\mathbf{6 b}-\mathrm{OTf}$ as a white powder. ${ }^{1} \mathrm{H}$ NMR (400 $\left.\mathrm{MHz}, \mathrm{CDCl}_{3}\right): \delta 1.33\left(\mathrm{~m}, 36 \mathrm{H}, \mathrm{CH}\left(\mathrm{CH}_{3}\right)_{2}\right), 2.02$ (br, $4 \mathrm{H}, \mathrm{CH}_{2} \mathrm{CH}_{2}$ ), $2.84\left(\mathrm{~m}, 6 \mathrm{H}, \mathrm{CH}\left(\mathrm{CH}_{3}\right)_{2}\right), 3.76\left(\mathrm{t}, J=7.0 \mathrm{~Hz}, 4 \mathrm{H}, \mathrm{NCH}_{2}\right), 4.15$ $\left(\mathrm{s}, 3 \mathrm{H}, \mathrm{OCH}_{3}\right) \cdot{ }^{13} \mathrm{C} \mathrm{NMR}\left(100 \mathrm{MHz}, \mathrm{CDCl}_{3}\right): \delta 19.9\left(\mathrm{CH}\left(\mathrm{CH}_{3}\right)_{2}\right)$, $24.7\left(\mathrm{t},{ }^{1} J_{\mathrm{PC}}=11.1 \mathrm{~Hz}, \mathrm{CH}\left(\mathrm{CH}_{3}\right)_{2}\right), 24.3\left(\mathrm{CH}_{2}\right), 24.5\left(\mathrm{CH}_{2}\right), 49.3$ $\left(\mathrm{NCH}_{2}\right), 51.8\left(\mathrm{NCH}_{2}\right), 60.7\left(\mathrm{OCH}_{3}\right), 95.3\left(\mathrm{t},{ }^{3} J_{\mathrm{PC}}=3.8 \mathrm{~Hz}, \mathrm{C}_{\beta}\right)$, $120.8\left(\mathrm{q},{ }^{1} J_{\mathrm{CF}}=320.0 \mathrm{~Hz}, \mathrm{CF}_{3}\right), 147.1\left(\mathrm{t},{ }^{2} J_{\mathrm{PC}}=11.6 \mathrm{~Hz}, \mathrm{C}_{\alpha}\right)$, $151.1\left(\mathrm{C}_{\gamma}\right) .{ }^{31} \mathrm{P}$ NMR $\left(162 \mathrm{MHz}, \mathrm{CDCl}_{3}\right): \delta$ 45.1. IR $\left(\mathrm{CH}_{2} \mathrm{Cl}_{2}\right)$ : $v(\mathrm{CCC}) 2086 \mathrm{~cm}^{-1} ; v(\mathrm{CO}) 1612 \mathrm{~cm}^{-1}$. UV-vis $\left(\mathrm{CH}_{2} \mathrm{Cl}_{2}\right): \lambda_{\max }(\mathrm{nm})$ $(\log \epsilon) 280$ (4.365). FAB-MS: $m / z(\%) 644(70)\left[(\mathrm{M}-\mathrm{OTf})^{+}\right]$, 483 (65) [(M - OTf $\left.\left.-\mathrm{PPh}_{3}\right)^{+}\right], 403$ (13) [(M - OTf - $\mathrm{PPh}_{3}-$ $\mathrm{Br})^{+}$]. Anal. Calcd for $\mathrm{C}_{27} \mathrm{H}_{53} \mathrm{BrF}_{3} \mathrm{NO}_{4} \mathrm{P}_{2} \mathrm{PdS}$ (793.05): C, 40.89; H, 6.74; N, 1.77. Found: C, 40.85; H, 7.19; N, 1.54.

trans-Bromobis[tris(4-methoxyphenyl)phosphine](3-,$N$-tetramethyleneamino-3-oxy-1-propynyl)palladium(II) (7b). The synthesis of $7 \mathbf{b}$ from $0.42 \mathrm{~g}(0.50 \mathrm{mmol})$ of $\mathbf{2 b}$ and $0.39 \mathrm{~g}(1.10 \mathrm{mmol}, 2.2$ equiv) of $\mathrm{P}\left(\mathrm{C}_{6} \mathrm{H}_{4} \mathrm{OMe}-4\right)_{3}$ in $30 \mathrm{~mL}$ of $\mathrm{CH}_{2} \mathrm{Cl}_{2}$ was carried out analogously to $\mathbf{5 a}$. The crude product was purified by column chromatography using a petroleum ether/ $\mathrm{CH}_{2} \mathrm{Cl}_{2}$ /acetone mixture. Yield: $0.37 \mathrm{~g}(0.37 \mathrm{mmol} ; 74 \%)$ of $\mathbf{7 b}$ as a pale yellow powder. ${ }^{1} \mathrm{H}$ NMR (400 MHz, $\left.\mathrm{CDCl}_{3}\right): \delta 1.40\left(\mathrm{~m}, 2 \mathrm{H}, \mathrm{CH}_{2}\right), 1.58(\mathrm{~m}, 2 \mathrm{H}$, $\left.\mathrm{CH}_{2}\right), 2.32\left(\mathrm{t}, J=6.8 \mathrm{~Hz}, 2 \mathrm{H}, \mathrm{NCH}_{2}\right), 3.06(\mathrm{t}, J=6.6 \mathrm{~Hz}, 2 \mathrm{H}$, $\left.\mathrm{NCH}_{2}\right), 3.78\left(\mathrm{~s}, 18 \mathrm{H}, \mathrm{OCH}_{3}\right), 6.85-6.87(\mathrm{~m}, 12 \mathrm{H}, \mathrm{ArH}), 7.57-7.61$ $(\mathrm{m}, 12 \mathrm{H}, \mathrm{ArH}) .{ }^{13} \mathrm{C} \mathrm{NMR}\left(100 \mathrm{MHz}, \mathrm{CDCl}_{3}\right): \delta 24.5\left(\mathrm{CH}_{2}\right), 24.9$ $\left(\mathrm{CH}_{2}\right), 44.1\left(\mathrm{NCH}_{2}\right), 47.0\left(\mathrm{NCH}_{2}\right), 55.1\left(\mathrm{OCH}_{3}\right), 104.5\left(\mathrm{t},{ }^{3} J_{\mathrm{PC}}=\right.$ $3.5 \mathrm{~Hz}, \mathrm{Pd}-\mathrm{C} \equiv C), 109.2\left(\mathrm{t},{ }^{3} J_{\mathrm{PC}}=9.0 \mathrm{~Hz}, \mathrm{Pd}-C \equiv \mathrm{C}\right), 113.5$ $(\mathrm{ArC}), 122.7\left(\mathrm{t},{ }^{2} J_{\mathrm{PC}}=27.8 \mathrm{~Hz}, \mathrm{ArC}\right), 136.3(\mathrm{ArC}), 153.1$ $\left(C(\mathrm{O}) \mathrm{NC}_{4} \mathrm{H}_{8}\right), 161.1$ (ArC). ${ }^{31} \mathrm{P}$ NMR $\left(162 \mathrm{MHz}, \mathrm{CDCl}_{3}\right): \delta 20.2$. IR $\left(\mathrm{CH}_{2} \mathrm{Cl}_{2}\right): v(\mathrm{CCC}) 2114 \mathrm{~cm}^{-1}$. UV-vis $\left(\mathrm{CH}_{2} \mathrm{Cl}_{2}\right): \lambda_{\max }(\mathrm{nm})(\log$ є) 317 (4.420). FAB-MS: m/z (\%) $1013(20)\left[\mathrm{M}^{+}\right], 933$ (85) [(M $\left.-\mathrm{Br})^{+}\right], 580(27)\left[\left(\mathrm{M}-\mathrm{Br}-\mathrm{P}\left(\mathrm{C}_{6} \mathrm{H}_{4} \mathrm{OMe}-p\right)_{3}\right)^{+}\right]$. Anal. Calcd for $\mathrm{C}_{49} \mathrm{H}_{50} \mathrm{BrNO}_{7} \mathrm{P}_{2} \mathrm{P} \mathrm{d} \times 0.5 \mathrm{CH}_{2} \mathrm{Cl}_{2}$ (1013.21): C, 56.32; $\mathrm{H}, 4.87$; $\mathrm{N}, 1,33$. Found: C, 55.69; H, 4.86; N, 1.02 .

trans-Bromobis[tris(4-methoxyphenyl)phosphine](3- $\mathrm{N}, \mathrm{N}$-tetramethyleneamino-3-methoxy-1,2-propadienylidene)palladium(II) Trifluoromethanesulfonate (8b-OTf). The synthesis of 8b-OTf from $0.58 \mathrm{~g}(0.57 \mathrm{mmol})$ of $\mathbf{7 b}$ and $0.07 \mathrm{~mL}(0.57 \mathrm{mmol})$ of MeOTf in $20 \mathrm{~mL}$ of $\mathrm{CH}_{2} \mathrm{Cl}_{2}$ was carried out analogously to 3a-OTf. Yield: $0.62 \mathrm{~g}(0.52 \mathrm{mmol} ; 91 \%)$ of $\mathbf{8 b}-\mathrm{OTf}$ as a yellow powder. ${ }^{1} \mathrm{H}$ NMR (400 MHz, $\left.\mathrm{CDCl}_{3}\right): \delta 1.68\left(\mathrm{~m}, 2 \mathrm{H}, \mathrm{CH}_{2}\right), 1.85\left(\mathrm{~m}, 2 \mathrm{H}, \mathrm{CH}_{2}\right), 2.72$ $\left(\mathrm{m}, 2 \mathrm{H}, \mathrm{NCH}_{2}\right), 3.35\left(\mathrm{~m}, 2 \mathrm{H}, \mathrm{NCH}_{2}\right), 3.78\left(\mathrm{~s}, 3 \mathrm{H}, \mathrm{OCH}_{3}\right), 3.81$ (s, $\left.18 \mathrm{H}, \mathrm{OCH}_{3}\right), 6.92-6.95(\mathrm{~m}, 12 \mathrm{H}, \mathrm{ArH}), 7.52-7.57(\mathrm{~m}, 12 \mathrm{H}$, ArH). $\left.{ }^{13} \mathrm{C} \mathrm{NMR} \mathrm{(100} \mathrm{MHz,} \mathrm{CDCl}_{3}\right): \delta 24.1\left(\mathrm{CH}_{2}\right), 24.4\left(\mathrm{CH}_{2}\right)$, $48.7\left(\mathrm{NCH}_{2}\right), 51.3\left(\mathrm{NCH}_{2}\right), 55.4\left(\mathrm{OCH}_{3}\right), 60.5\left(\mathrm{OCH}_{3}\right), 93.9\left(\mathrm{C}_{\beta}\right)$, $114.0(\mathrm{ArC}), 121.6\left(\mathrm{t},{ }^{1} J_{\mathrm{PC}}=28.7 \mathrm{~Hz}, \mathrm{ArC}\right), 136,3\left(\mathrm{t},{ }^{2} J_{\mathrm{PC}}=7.1\right.$ $\mathrm{Hz}, \mathrm{ArC}), 151.1\left(\mathrm{C}_{\gamma}\right), 161.8(\mathrm{ArC}), 177.0\left(\mathrm{C}_{\alpha}\right) \cdot{ }^{31} \mathrm{P}$ NMR $(162 \mathrm{MHz}$, $\left.\mathrm{CDCl}_{3}\right): \delta$ 20.4. ${ }^{19} \mathrm{~F} \mathrm{NMR}\left(376 \mathrm{MHz}, \mathrm{CDCl}_{3}\right): \delta$-78.2. IR $\left(\mathrm{CH}_{2} \mathrm{Cl}_{2}\right): v(\mathrm{CCC}) 2098 \mathrm{~cm}^{-1}$. UV-vis $\left(\mathrm{CH}_{2} \mathrm{Cl}_{2}\right): \lambda_{\max }(\mathrm{nm})(\log$ є) 328 (4.352). FAB-MS: $m / z$ (\%) $1028(8)\left[(\mathrm{M}-\mathrm{OTf})^{+}\right], 948(8)$ $\left[(\mathrm{M}-\mathrm{OTf}-\mathrm{Br})^{+}\right], 595(23)\left[\left(\mathrm{M}-\mathrm{OTf}-\mathrm{Br}-\mathrm{P}\left(\mathrm{C}_{6} \mathrm{H}_{4} \mathrm{OMe}-\right.\right.\right.$ $\left.p)_{3}\right)^{+}$. Anal. Calcd for $\mathrm{C}_{51} \mathrm{H}_{53} \mathrm{BrF}_{3} \mathrm{NO}_{10} \mathrm{P}_{2} \mathrm{PdS}$ (1177.32): C, 52.03; H, 4.54; N, 1.19. Found: C, 52.10; H, 4.72; N, 1.26.

trans-(Trifluoroacetato)bis(triphenylphosphine)(3-dimethylamino3-oxy-1-propynyl)palladium(II) (9a). A suspension of $0.55 \mathrm{~g}(0.68$ $\mathrm{mmol})$ of $2 \mathrm{a}$ and $0.15 \mathrm{~g}(0.68 \mathrm{mmol})$ of $\mathrm{CF}_{3} \mathrm{COOAg}$ in $30 \mathrm{~mL}$ of dry $\mathrm{CH}_{2} \mathrm{Cl}_{2}$ was stirred for $30 \mathrm{~min}$. The precipitate $(\mathrm{AgBr})$ that formed was filtered off. The solvent of the crude reaction mixture was removed in vacuo. Crystallization of the crude product from $\mathrm{CH}_{2} \mathrm{Cl}_{2} / \mathrm{Et}_{2} \mathrm{O}$ gave $0.54 \mathrm{~g}(0.65 \mathrm{mmol} ; 96 \%)$ of $\mathbf{9 a}$ as a colorless powder. ${ }^{1} \mathrm{H}$ NMR (400 MHz, $\mathrm{CD}_{2} \mathrm{Cl}_{2}$ ): $\delta 1.93\left(\mathrm{~s}, 3 \mathrm{H}, \mathrm{NCH}_{3}\right), 2.47$ (s, 3H, $\left.\mathrm{NCH}_{3}\right), 7.41-7.51(\mathrm{~m}, 18 \mathrm{H}, \mathrm{ArH}), 7.71-7.79(\mathrm{~m}, 12 \mathrm{H}$, ArH). ${ }^{13} \mathrm{C} \mathrm{NMR}\left(100 \mathrm{MHz}, \mathrm{CD}_{2} \mathrm{Cl}_{2}\right): \delta 33.2\left(\mathrm{NCH}_{3}\right), 37.2\left(\mathrm{NCH}_{3}\right)$, $93.8\left(\mathrm{t},{ }^{2} J_{\mathrm{PC}}=10.7 \mathrm{~Hz}, \mathrm{Pd}-C \equiv \mathrm{C}\right), 107.4(\mathrm{Pd}-\mathrm{C} \equiv C), 107.4$ $\left(\mathrm{CF}_{3} \mathrm{COO}\right), 128.4\left(\mathrm{t},{ }^{3} J_{\mathrm{PC}}=5.7 \mathrm{~Hz}, m-\mathrm{C}\right), 129.6\left(\mathrm{t},{ }^{1} J_{\mathrm{PC}}=25.0\right.$ $\mathrm{Hz}, i-\mathrm{C}), 131.2(p-\mathrm{C}), 134.1\left(\mathrm{t},{ }^{2} J_{\mathrm{PC}}=5.3 \mathrm{~Hz}, o-\mathrm{C}\right), 154.3$ $\left(C(\mathrm{O}) \mathrm{NMe}_{2}\right), 174.3\left(\mathrm{CF}_{3} \mathrm{COO}\right) .{ }^{31} \mathrm{P}$ NMR $\left(162 \mathrm{MHz}, \mathrm{CD}_{2} \mathrm{Cl}_{2}\right): \delta$ 23.9. IR (THF): $v(\mathrm{C} \equiv \mathrm{C}) 2114 \mathrm{~cm}^{-1} ; v(\mathrm{CO}) 1680,1609 \mathrm{~cm}^{-1}$. $\mathrm{UV}-\mathrm{vis}\left(\mathrm{CH}_{2} \mathrm{Cl}_{2}\right): \lambda_{\max }(\mathrm{nm})(\log \epsilon) 239$ (4.513), 297 (4.449). FABMS: $m / z(\%) 727(11)\left[\left(\mathrm{M}-\mathrm{CF}_{3} \mathrm{COO}\right)^{+}\right], 631(62)\left[\left(\mathrm{M}-\mathrm{CF}_{3} \mathrm{COO}\right.\right.$ $\left.-\mathrm{C}_{5} \mathrm{H}_{6} \mathrm{NO}\right)^{+}$], $369(62)\left[\left(\mathrm{M}-\mathrm{CF}_{3} \mathrm{COO}-\mathrm{C}_{5} \mathrm{H}_{6} \mathrm{NO}-\mathrm{PPh}_{3}\right)^{+}\right]$. Anal. Calcd for $\mathrm{C}_{43} \mathrm{H}_{36} \mathrm{~F}_{3} \mathrm{NO}_{3} \mathrm{P}_{2} \mathrm{Pd} \cdot \mathrm{CH}_{2} \mathrm{Cl}_{2}$ (840.13): $\mathrm{C}$, 57.13; $\mathrm{H}$, 4.14 ; N, 1.51. Found: C, 57.52; H, 4.30; N, 1.81 .

trans-(Trifluoroacetato)bis(triphenylphosphine)(3-dimethylamino3-methoxy-1,2-propadienylidene)palladium(II) Trifluoromethanesulfonate (10a-OTf). The synthesis of 10a-OTf from $0.49 \mathrm{~g}(0.58$ $\mathrm{mmol})$ of $9 \mathbf{a}$ and $0.07 \mathrm{~mL}(0.62 \mathrm{mmol})$ of MeOTf in $30 \mathrm{~mL}$ of $\mathrm{CH}_{2} \mathrm{Cl}_{2}$ was carried out analogously to 3a-OTf. Recrystallization from mixtures of $\mathrm{CH}_{2} \mathrm{Cl}_{2}$ and pentane afforded $0.52 \mathrm{~g}(0.52 \mathrm{mmol}$, $90 \%$ ) of pure 10a-OTf as a colorless powder. ${ }^{1} \mathrm{H}$ NMR $(400 \mathrm{MHz}$, $\left.\mathrm{CD}_{2} \mathrm{Cl}_{2}\right): \delta 2.46\left(\mathrm{~s}, 3 \mathrm{H}, \mathrm{NCH}_{3}\right), 2.80\left(\mathrm{~s}, 3 \mathrm{H}, \mathrm{NCH}_{3}\right), 3.03(\mathrm{~s}, 3 \mathrm{H}$, $\left.\mathrm{OCH}_{3}\right), 7.38-7.47$ (m, 18H, ArH), 7.58-7.65 (m, 12H, ArH). ${ }^{13} \mathrm{C}$ NMR $\left(100 \mathrm{MHz}, \mathrm{CD}_{2} \mathrm{Cl}_{2}\right): \delta 38.0\left(\mathrm{NCH}_{3}\right), 41.5\left(\mathrm{NCH}_{3}\right), 61.2$ $\left(\mathrm{OCH}_{3}\right), 95.6\left(\mathrm{C}_{\beta}\right), 110.9\left(\mathrm{CF}_{3} \mathrm{COO}\right), 120.0\left(\mathrm{SO}_{3} \mathrm{CF}_{3}\right), 128.3\left(\mathrm{t},{ }^{1} J_{\mathrm{PC}}\right.$ $=25.8 \mathrm{~Hz}, i-\mathrm{C}), 129.3\left(\mathrm{t},{ }^{3} J_{\mathrm{PC}}=5.8 \mathrm{~Hz}, m-\mathrm{C}\right), 132.1(p-\mathrm{C}), 134.7$ $\left(\mathrm{t},{ }^{2} J_{\mathrm{PC}}=6.7 \mathrm{~Hz}, o-\mathrm{C}\right), 137.9\left(\mathrm{t},{ }^{3} J_{\mathrm{PC}}=5.7 \mathrm{~Hz}, \mathrm{C}_{\alpha}\right), 153.7\left(\mathrm{C}_{\gamma}\right)$, $172.8\left(\mathrm{CF}_{3} \mathrm{COO}\right) .{ }^{31} \mathrm{P}$ NMR (162 MHz, $\left.\mathrm{CD}_{2} \mathrm{Cl}_{2}\right): \delta 25.9$. IR (THF): $v(\mathrm{CCC}) 2102 \mathrm{~cm}^{-1} ; v(\mathrm{CO}) 1678 \mathrm{~cm}^{-1}$. UV-vis $\left(\mathrm{CH}_{2} \mathrm{Cl}_{2}\right): \lambda_{\max }(\mathrm{nm})$ (log $\epsilon) 251$ (4.359), 307 (4.528). FAB-MS: $m / z$ (\%) 855 (13) [(M - OTf $\left.)^{+}\right], 592(80)\left[\left(\mathrm{M}-\mathrm{OTf}-\mathrm{PPh}_{3}\right)^{+}\right], 478$ (39) [(M - OTf $\left.\left.\mathrm{PPh}_{3}-\mathrm{CF}_{3} \mathrm{COO}\right)^{+}\right]$. Anal. Calcd for $\mathrm{C}_{45} \mathrm{H}_{39} \mathrm{~F}_{6} \mathrm{NO}_{6} \mathrm{P}_{2-}$ $\mathrm{PdS} \cdot 0.5 \mathrm{CH}_{2} \mathrm{Cl}_{2}$ (1004.23): C, 52.21; H, 3.85; N, 1.34. Found: $\mathrm{C}$, 51.94; H, 4.07; N, 1.44 .

trans-(Trifluoracetato)bis(triphenylphosphine)(3-dimethylamino3-methoxy-1,2-propadienylidene)palladium(II) Tetrafluoroborate $\left(\mathbf{1 0 a}-\mathbf{B F}_{\mathbf{4}}\right)$. The synthesis of $\mathbf{1 0 a}-\mathrm{BF}_{4}$ from $0.47 \mathrm{~g}(0.56 \mathrm{mmol})$ of 9a and $99 \mathrm{mg}\left(0.67 \mathrm{mmol}, 1.2\right.$ equiv) of $\left[\mathrm{Me}_{3} \mathrm{O}\right] \mathrm{BF}_{4}$ in $30 \mathrm{~mL}$ of 
$\mathrm{CH}_{2} \mathrm{Cl}_{2}$ was carried out analogously to 3a-OTf. Yield: $0.45 \mathrm{~g}(0.48$ mmol; $86 \%)$ of $\mathbf{1 0 a}-\mathrm{BF}_{4}$ as an off-white powder. ${ }^{1} \mathrm{H}$ NMR (400 $\left.\mathrm{MHz}, \mathrm{CDCl}_{3}\right): \delta 2.55\left(\mathrm{~s}, 3 \mathrm{H}, \mathrm{NCH}_{3}\right), 2.89\left(\mathrm{~s}, 3 \mathrm{H}, \mathrm{NCH}_{3}\right), 3.10$ (s, $\left.3 \mathrm{H}, \mathrm{OCH}_{3}\right), 7.49-7.57$ (m, $\left.18 \mathrm{H}, \mathrm{ArH}\right), 7.68-7.72(\mathrm{~m}, 12 \mathrm{H}$, ArH). ${ }^{13} \mathrm{C}$ NMR $\left(100 \mathrm{MHz}, \mathrm{CD}_{2} \mathrm{Cl}_{2}\right): \delta 38.0\left(\mathrm{NCH}_{3}\right), 41.7\left(\mathrm{NCH}_{3}\right)$, $61.3\left(\mathrm{OCH}_{3}\right), 128.4\left(\mathrm{t},{ }^{1} J_{\mathrm{PC}}=25.9 \mathrm{~Hz}, i-\mathrm{C}\right), 129.4\left(\mathrm{t},{ }^{3} J_{\mathrm{PC}}=5.7\right.$ $\mathrm{Hz}, \mathrm{m}-\mathrm{C}), 132.2$ (p-C), 134.9 (t, $\left.{ }^{2} J_{\mathrm{PC}}=6.4 \mathrm{~Hz}, o-\mathrm{C}\right), 153.8\left(\mathrm{C}_{\gamma}\right)$; $\mathrm{C}_{\alpha}, \mathrm{C}_{\beta}, \mathrm{CF}_{3} \mathrm{COO}$ not observed. ${ }^{31} \mathrm{P}$ NMR $\left(162 \mathrm{MHz}, \mathrm{CD}_{2} \mathrm{Cl}_{2}\right): \delta$ 26.2. IR (THF): $v$ (CCC) $2103 \mathrm{~cm}^{-1} ; v(\mathrm{CO}) 1678 \mathrm{~cm}^{-1}$. UV-vis $\left(\mathrm{CH}_{2} \mathrm{Cl}_{2}\right): \lambda_{\max }(\mathrm{nm})(\log \epsilon) 307$ (4.450). FAB-MS: $\mathrm{m} / \mathrm{z}$ (\%) 854 (33) $\left[\left(\mathrm{M}-\mathrm{BF}_{4}\right)^{+}\right], 663(100)\left[\left(\mathrm{M}-\mathrm{BF}_{4}-\mathrm{CF}_{3} \mathrm{COO}-\mathrm{Ph}\right)^{+}\right]$, 631 (49) $\left[\left(\mathrm{M}-\mathrm{BF}_{4}-\mathrm{CF}_{3} \mathrm{COO}-\mathrm{CCC}(\mathrm{OMe}) \mathrm{NMe}^{2}\right)^{+}\right], 592(68)$ $\left[\left(\mathrm{M}-\mathrm{BF}_{4}-\mathrm{PPh}^{3}\right)^{+}\right], 479(37)\left[\left(\mathrm{M}-\mathrm{BF}_{4}-\mathrm{PPh}_{3}-\mathrm{CF}_{3} \mathrm{COO}\right)^{+}\right]$, 369 (82) $\left[\left(\mathrm{M}-\mathrm{BF}_{4}-\mathrm{PPh}_{3}-\mathrm{CF}_{3} \mathrm{COO}-\mathrm{CCC}(\mathrm{OMe}) \mathrm{NMe}_{2}\right)^{+}\right]$. Anal. Calcd for $\mathrm{C}_{44} \mathrm{H}_{39} \mathrm{BF}_{7} \mathrm{NO}_{3} \mathrm{P}_{2} \mathrm{Pd}$ (941.96): C, 56.10; H, 4.17; N, 1.49. Found: 56.03; H, 4.22; N, 1.42 .

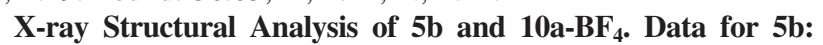
$\mathrm{C}_{25} \mathrm{H}_{50} \mathrm{BrNOP} 2 \mathrm{Pd} \cdot \mathrm{CDCl}_{3}, M_{\mathrm{r}}=748.28$, monoclinic, space group $P 2{ }_{1} / c, a=8.9048(18) \AA, b=11.445(2) \AA, c=32.830(7) \AA, \beta=$ $91.24(3)^{\circ}, V=3345.1(12) \AA^{3}, Z=4, d_{\text {calcd }}=1.486 \mathrm{~g} \mathrm{~cm}^{-3}, F(000)$ $=1536, \mu=2.104 \mathrm{~mm}^{-1}, 2 \theta_{\max }=51.3^{\circ}$, index ranges $-10 \leq h$ $\leq 10,-13 \leq k \leq 13,-39 \leq l \leq 39,36114$ data (6273 unique), $R($ int $)=0.0931,319$ parameters, R1 $(I>2 \sigma(I))=0.0343$, wR2 $=0.0776$, goodness of fit on $F^{2} 1.042, \Delta \rho_{\max }\left(\Delta \rho_{\min }\right)=0.649$ $(-0.855)$ e $\AA^{-3}$.

Data for 10a-BF : $_{46} \mathrm{H}_{43} \mathrm{BCl}_{4} \mathrm{~F}_{7} \mathrm{NO}_{3} \mathrm{P}_{2} \mathrm{Pd}, M_{\mathrm{r}}=1111.76$, monoclinic, space group $P 2_{1} / n, a=11.612(2) \AA, b=23.143(5) \AA, c=$ 18.136(4) $\AA, \beta=90.71(3)^{\circ}, V=4873.5(17) \AA^{3}, Z=4, d_{\text {calcd }}=$
$1.515 \mathrm{~g} \mathrm{~cm}^{-3}, F(000)=2248, \mu=0.733 \mathrm{~mm}^{-1}, 2 \theta_{\max }=53.7^{\circ}$, index ranges $-14 \leq h \leq 14,-29 \leq k \leq 29,-23 \leq l \leq 22,70118$ data: (10 300 unique), $R$ (int) $=0.0879,586$ parameters, R1 $(I>$ $2 \sigma(I))=0.0406$, wR2 $=0.0959$, goodness of fit on $F^{2} 1.023, \Delta \rho_{\max }$ $\left(\Delta \rho_{\min }\right)=1.132(-0.975)$ e $\AA^{-3}$.

Single crystals suitable for an $\mathrm{X}$-ray structural analysis of $\mathbf{5 b}$ were grown from $\mathrm{CDCl}_{3}$ and those of $\mathbf{1 0 a}-\mathrm{BF}_{4}$ by slow diffusion of hexane into a concentrated solution of $\mathbf{1 0 a}-\mathrm{BF}_{4}$ in $\mathrm{CH}_{2} \mathrm{Cl}_{2}$ at 4 ${ }^{\circ} \mathrm{C}$. The measurements were performed at $100(2) \mathrm{K}$ with a crystal mounted on a glass fiber on a Stoe IPDS II diffractometer (graphite monochromator, Mo K $\alpha$ radiation, $\lambda=0.71073 \AA$ ). The structures were solved by direct methods using the SHELX-97 program package. ${ }^{21}$ The positions of the hydrogen atoms were calculated by assuming ideal geometry, and their coordinates were refined together with those of the attached carbon atoms as the riding model. All other atoms were refined anisotropically.

Acknowledgment. Support of this work by the WackerChemie GmbH (gift of chemicals) is gratefully acknowledged.

Supporting Information Available: CIF files of the complexes $\mathbf{5 b}$ and $\mathbf{1 0 a}-\mathrm{BF}_{4}$ and tables giving the bond distances, bond angles, and torsion angles of $\mathbf{5 b}$ and $\mathbf{1 0 a}-\mathrm{BF}_{4}$. This material is available free of charge via the Internet at http://pubs.acs.org.

\section{OM800843E}

(21) Sheldrick, G. M. SHELXTL-97, Programs for Crystal Structure Analysis; University of Göttingen, Göttingen, Germany, 1997. 